Portland State University

PDXScholar

8-29-1997

\title{
Symmetry, Symmetry Breaking, and the Current View of the Dirac Monopole
}

Erik Kristian Leder

Portland State University

Follow this and additional works at: https://pdxscholar.library.pdx.edu/open_access_etds

Part of the Physics Commons

Let us know how access to this document benefits you.

\section{Recommended Citation}

Leder, Erik Kristian, "Symmetry, Symmetry Breaking, and the Current View of the Dirac Monopole" (1997). Dissertations and Theses. Paper 5392.

https://doi.org/10.15760/etd.7261

This Thesis is brought to you for free and open access. It has been accepted for inclusion in Dissertations and Theses by an authorized administrator of PDXScholar. Please contact us if we can make this document more accessible: pdxscholar@pdx.edu. 


\section{THESIS APPROVAL}

The abstract and thesis of Erik Kristian Leder for the Master of Science in

Physics were presented May 8, 1997, and accepted by the thesis committee and the department.

COMMITTEE APPROVALS:

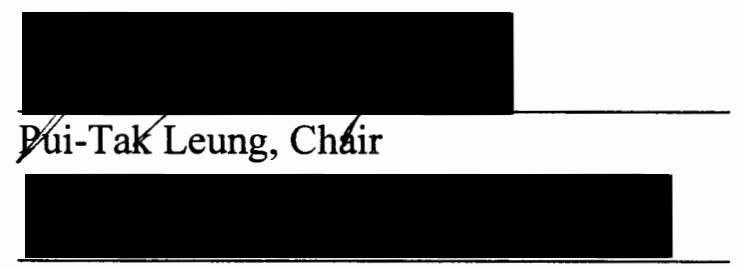

Frank Thiess

DEPARTMENT APPROVAL:
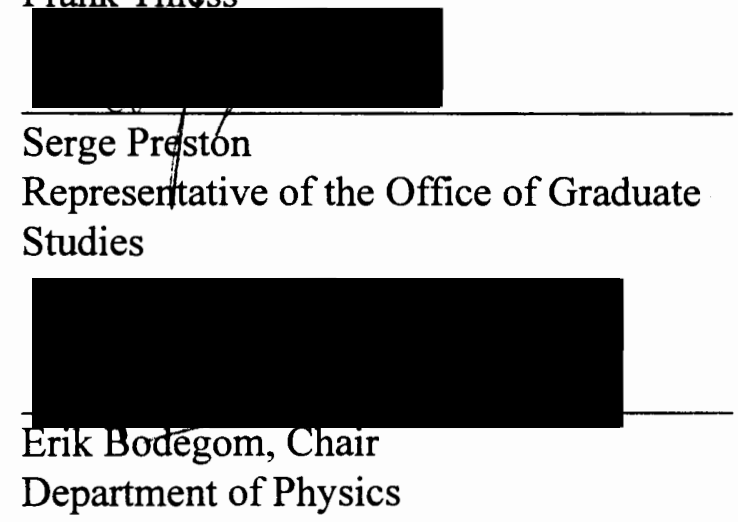

$* * * * * * * * * * * * * * * * * * * * * * * * * * * * * * * * * * * * * * * * * * * * * * * * * * * * * * * * * * * * * * *$

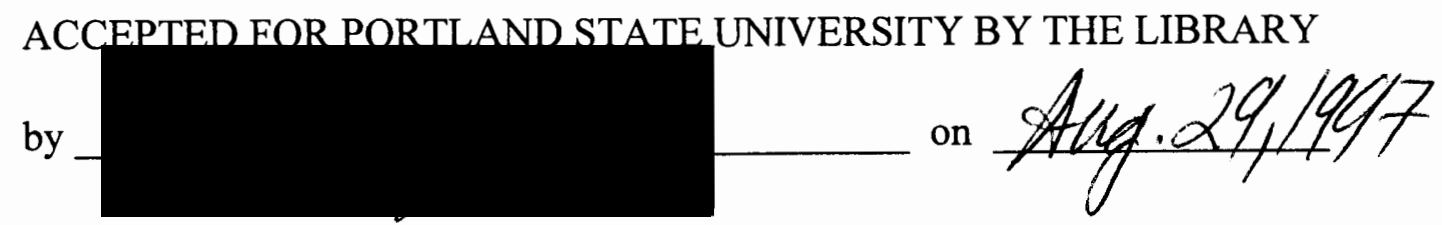




\begin{abstract}
An abstract of the thesis of Erik Kristian Leder for the Master of Science in Physics presented May 8, 1997.

Title: Symmetry, Symmetry Breaking, and the Current View of the Dirac Monopole.

The ideas of symmetry and symmetry breaking are considered in the context of classical gauge theory. Local U(1) symmetry is developed for a complex scalar field and electromagnetism is identified as the associated gauge field. Local $\mathrm{SO}(3)$ theory for a triplet of real scalar fields is derived and the incorporation of additional gauge fields is demonstrated. Symmetry breaking for the $\mathrm{SO}(3)$ gauge theory is performed and the gauge fields are shown to acquire mass by the Higgs mechanism. The equations of the t'Hooft-Polyakov monopole are derived and compared to those of the magnetic monopole of Dirac.
\end{abstract}




\begin{abstract}
SYMMETRY, SYMMETRY BREAKING, AND THE CURRENT VIEW OF THE DIRAC MONOPOLE
\end{abstract}

by

ERIK KRISTIAN LEDER

A thesis submitted in partial fulfillment of the requirements for the degree of

MASTER OF SCIENCE

in

PHYSICS

Portland State University

1997 


\section{CONTENTS}

1. Introduction and Background ............................................... 1

2. The Abelian Model and Electromagnetism .............................. 9

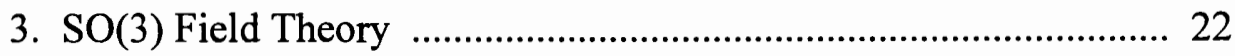

4. Symmetry Breaking ....................................................... 28

5. Finite Energy Solutions and the t'Hooft-Polyakov Monopole ...... 33

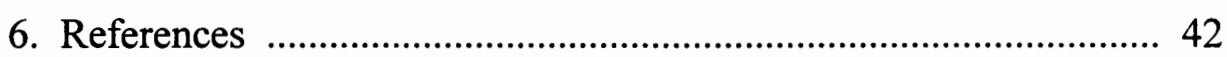




\section{Introduction and Background}

"The steady progress of physics requires for its theoretical formulation a mathematics that gets continually more advanced. This is only natural and to be expected. What, however, was not expected by the scientific workers of the last century was the particular form that the line of advancement would take, namely, it was expected that the mathematics would get more and more complicated, but would rest on a permanent basis of axioms and definitions, while actually the modern physical developments have required a mathematics that continually shifts its foundations and gets more abstract. Non-euclidean geometry and non-commutative algebra, which were at one time considered to be purely fictions of the mind and pastimes for logical thinkers, have now been found to be very necessary for the description of general facts of the physical world. It seems likely that this process of increasing abstraction will continue in the future and that advances in physics is to be associated with a continual modification and generalisation of the axioms at the base of mathematics rather than with a logical development of any one mathematical scheme on a fixed foundation."

- P.A.M. Dirac, "Quantised Singularities in the Electromagnetic Field", 1931

\subsection{Introduction}

The purpose of this paper is to understand the current view of the magnetic monopole in the context of gauge field theory. A major portion of this paper focuses on developing some of the fundamental concepts and mathematical methods that lie at the foundation of the current approach to elementary particle physics. Some basic features of gauge theory are first developed using the U(1) symmetry, which underlies electrodynamics. The concepts presented in this simple setting are 
then generalized to a non-abelian model using $\mathrm{SO}(3)$ as the symmetry group. When this symmetry is then broken we find as a residue the $U(1)$ symmetry of electromagnetism. An important solution to the broken $\mathrm{SO}(3)$ symmetry field equations exhibit at large distances all the properties of a magnetic monopole.

In 1931 Dirac published the above-quoted paper [1] in which he considered the possibility of magnetic monopoles existing in nature. He was led to the concept of magnetic monopole through examining the indeterminacy and non-integrability of the phase of the quantum mechanical wave function. His analysis established the connection between invariance under phase change of quantum states and electromagnetism. In the process Dirac argued that the existence of magnetic monopoles leads to the quantization of electric charge. The argument concerning charge quantization may be carried out by examining the angular momentum of a charged particle in the presence of a magnetic monopole, as in Cheng and $\mathrm{Li}$ [2]. We briefly survey Dirac's original and elegant argument, since its pioneering ideas pertains to the concepts presented in this paper.

He begins by observing that the phase of the wave function is indeterminate everywhere and that the change in phase will have a well-defined difference only for neighboring, that is infinitesimally close, points. As a consequence, the change in phase around along closed curves will not vanish in general. Dirac argues that this non-integrability of phase can have no observable consequences and demonstrates that the change of phase is associated to interactions with electromagnetic fields.

It is possible for the phase difference around closed loops could differ for different wave functions by integral multiples of $2 \pi$, but if the loop is sufficiently small, the continuity of the wave function will guarantee that the phase difference remains small, and therefore equal, for all wave functions. The notable exception is when 
the wave function vanishes along a line (nodal singularity) that passes through the loop. Then, it is possible for the wave function to possess a phase difference that is undetermined up to $2 n \pi$ for some $\mathrm{n}$ regardless of the size of the loop. This integer, $\mathrm{n}$, characterizes the nodal line. The change in phase of any wave function around a small closed curve $\mathrm{C}$ encircling this nodal singularity can be expressed in terms of this integer. Furthermore, the change of phase for wave functions without a singular line passing through the curve $\mathrm{C}$ is given by some quantity of magnetic flux passing through the this loop. The change in phase for all wave functions is given by

$$
2 \pi n+\frac{e}{\hbar c} \int_{S} d \mathbf{S} \cdot \mathbf{H}
$$

where $\mathrm{S}$ is a surface with boundary $\mathrm{C}$ and $e$ is the electric charge.

Now, classically, the total magnetic flux normally vanishes for surfaces without boundary since $\nabla \cdot H=0$. But this last expression was found by quantum mechanical arguments. There is no a priori reason to assume that this integer must be zero. If $\mathrm{n}$ is not zero, then it follows that at least one of the singular line entering the surface terminates. The endpoint of this nodal line is a singularity in the electromagnetic field that corresponds to a source of magnetic flux. Furthermore, since this expression is not a property of any particular wave function, it holds true for all wave functions, and, consequently, all wave functions must possesses a singular line that terminates at this specific point, a magnetic monopole. The quantization condition follows by applying this last expression to closed surfaces without boundary. By Stoke's theorem, the change of phase must now vanish, so that the above integral is equal to zero for all such surfaces. If we enclose a single terminating point by such a surface,

$$
2 \pi n+\frac{e q_{M}}{\hbar c}=0
$$


where $q_{M}$ is the charge of the magnetic pole. The expression in (1.2) is the quantization condition.

To understand magnetic monopoles as viewed in the context of present-day theory, we first must develop the concepts of symmetry and symmetry-breaking in Lagrangian field theory. In Section 1.2 and 1.3, we collect for later reference, material that will be used throughout the rest of this paper. Section 2 is devoted to the abelian prototype of gauge field theory which recasts Dirac's indeterminacy of phase into the modern notion of $\mathrm{U}(1)$ invariance in the context of a complex scalar field. This section also includes a reformulation of this theory using representations of the isomorphic group $\mathrm{SO}(2)$. In Section 3, we generalize to the more complicated symmetry group $\mathrm{SO}(3)$. Spontaneous symmetry breaking will be discussed in Section 4 , where we will see how symmetry gives rise to degenerate vacuum configurations and how mass is acquired by the various field. Finally, in the section 5, we develop the equations of the t'Hooft-Polyakov monopole, which exhibit properties of the Dirac magnetic monopole.

\section{Section 1.2: Notation}

The field theories considered is this paper are defined on Minkowski space-time M. The metric

$$
\left(\eta_{\mu \nu}\right)=\left(\begin{array}{cccc}
1 & 0 & 0 & 0 \\
0 & -1 & 0 & 0 \\
0 & 0 & -1 & 0 \\
0 & 0 & 0 & -1
\end{array}\right)
$$

defines the invariant distance $d s^{2}=\eta_{\mu \nu} d x^{\mu} d x^{\nu}=d t^{2}-d x^{2}-d y^{2}-d z^{2}$, where the speed of light $\mathrm{c}=1$. A four-vector is a contravariant vector with components $v_{\mu}$, where $v_{0}$ is the time component and $v_{i}(\mathrm{i}=1,2,3)$ are the spatial components for the 4 -vector. The inner product of two 4 -vectors $\left(v_{\mu}\right)$ and $\left(w_{\mu}\right)$ is given by the usual 
expression

$$
v \cdot w=v^{\mu} w_{\mu}=v_{\mu} w^{\mu}
$$

where the covariant components are given by $v^{\mu}=\eta^{\mu \nu} v_{\nu}$.

The derivatives $\partial_{\mu}$ form a four-vector that has components

$$
\partial_{\mu}=\frac{\partial}{\partial x^{\mu}} \quad \mu=0,1,2,3
$$

Thus the four-gradient of a scalar field $\psi$ is the vector having components

$$
\partial_{\mu} \psi=\frac{\partial \psi}{\partial x^{\mu}}
$$

The d'Alembertian operator is formed by taking the inner product

$$
\square=\partial_{\mu} \cdot \partial_{\mu}=\frac{\partial^{2}}{\partial t^{2}}-\frac{\partial^{2}}{\partial x^{2}}-\frac{\partial^{2}}{\partial y^{2}}-\frac{\partial^{2}}{\partial z^{2}}
$$

\section{Section 1.3: Action Principles and Noether's Theorem}

The field theoretic version of the action principle [3] is a generalization of that found in ordinary mechanics. The action is a functional on the space of fields comprising the theory that has the general form

$$
S[\psi]=\int d t\{L\}=\int d^{4} x\left\{\mathcal{L}\left(\psi, \partial_{\mu} \psi\right)\right\}
$$

where $\mathcal{L}$ is the Lagrangian density of the theory. The action principle states that the evolution of the system represented by (1.8) corresponds to the stationary (minimum) values of $\mathrm{S}$ under an independent variation of the field components. This results in the Euler-Lagrange equations:

$$
\frac{\partial \mathcal{L}}{\partial \psi^{i}}-\partial_{\mu} \frac{\partial \mathcal{L}}{\partial\left(\partial_{\mu} \psi^{i}\right)}=0
$$

for $i=1, \ldots, N$ where $\mathrm{N}$ is the number of field components included in the theory. 
Generally, the action (1.8) will be invariant under a continuous group $G$ of transformations of the Noether's theorem provides a strong connection between the symmetries of the action $\mathrm{S}$ and conserved quantities along solutions of the EulerLagrange equations.

Invariance under space-time translations having the infinitesimal form

$$
\begin{aligned}
& x^{\mu} \rightarrow \tilde{x}^{\mu}=x^{\mu}+\delta_{\nu}^{\mu} \epsilon^{\nu} \\
& \psi^{i} \rightarrow \psi^{i}
\end{aligned}
$$

leads to the conserved energy-momentum tensor

$$
\mathcal{T}^{\mu}{ }_{\nu}=\sum_{i=1}^{N} \frac{\partial \mathcal{L}}{\partial\left(\partial_{\mu} \psi^{i}\right)} \partial_{\nu} \psi^{i}-\delta_{\nu}^{\mu} \mathcal{L}
$$

satisfying $\partial_{\mu} \mathcal{T}^{\mu}{ }_{\nu}=0$ for solutions of the field equations (1.9). The symmetries associated with the internal space of the theory also result in conserved currents. Under an infinitesimal transformation of the field

$$
\begin{aligned}
& x^{\mu} \rightarrow x^{\mu} \\
& \psi^{i} \rightarrow \psi^{i}+\Omega_{a}^{i} \theta^{a},
\end{aligned}
$$

where $a$ index the various independent generators of the symmetry group $G$. The conserved Noether currents are

$$
j_{\alpha}^{\mu}=-\sum_{i=1}^{N} \frac{\partial \mathcal{L}}{\partial\left(\partial_{\mu} \psi^{i}\right)} \Omega^{i} \alpha \quad \alpha=1, \ldots, m
$$

where the $\Omega$ terms contain information about the $m$ generators of the symmetry group. The currents are conserved, in the sense that $\partial_{\mu} j_{\alpha}^{\mu}=0$, along the solutions to the Euler-Lagrange equations. If, in addition, the theory contains objects with multiple indices, like gauge fields, then (1.13) generalizes to

$$
j_{\alpha}^{\mu}=-\sum_{i=1}^{N}\left\{\frac{\partial \mathcal{L}}{\partial\left(\partial_{\mu} \psi^{i}\right)} \Omega_{\alpha}^{i}+\frac{\partial \mathcal{L}}{\partial\left(\partial_{\mu} A_{k}^{i}\right)} \Omega_{k \alpha}^{i}\right\}
$$




\section{4: Equivalent Forms of the Maxwell Equations}

Maxwell's equations in vector form are given by

$$
\begin{array}{ll}
\nabla \cdot \mathbf{E}=\rho, & \nabla \times \mathbf{B}-\partial_{0} \mathbf{E}=\mathbf{j}, \\
\nabla \cdot \mathbf{B}=0, & \nabla \times \mathbf{E}+\partial_{0} \mathbf{B}=0
\end{array}
$$

where $\rho$ and $\mathbf{j}$ are the charge and current densities, respectively. The equation $\nabla \cdot \mathrm{B}=0$ expresses the absence of magnetic sources in classical electrodynamics. The electric and magnetic fields in (1.15) and (1.16) can be expressed in terms of the scalar potential $\phi$ and the vector potential $\mathbf{A}$ by

$$
\mathbf{E}=-\nabla \phi-\partial_{0} \mathbf{A}, \quad \mathbf{B}=\nabla \times \mathbf{A}
$$

The equations (1.16) are transcribed into tensor form by the following identifications [4]. The components of the antisymmetric field strength tensor are given by

$$
\mathcal{F}^{0 i}=E^{i} \quad i=1,2,3
$$

and

$$
\mathcal{F}^{i j}=\epsilon^{i j k} B^{k} \quad i, j=1,2,3 .
$$

The charge and current densities form the time and spatial components, respectively, of the four-current:

$$
\left(j^{\mu}\right)=(\rho, \mathbf{j})
$$

Furthermore, the components of the field strength can be expressed in terms of the four-potential

$$
\left(A_{\mu}\right)=(\phi, \mathbf{A})
$$


by

$$
\mathcal{F}_{\mu \nu}=\partial_{\mu} A_{\nu}-\partial_{\nu} A_{\mu}
$$

The field strength $\mathcal{F}_{\mu \nu}$ by itself generates one pair of Maxwell's equation. The second pair follows from the dual tensor $\tilde{\mathcal{F}}$ with components defined by

$$
\tilde{\mathcal{F}}^{\mu \nu}=\frac{1}{2} \epsilon^{\mu \nu \sigma \tau} \mathcal{F}_{\sigma \tau}
$$

The equations (1.16) can be then written in terms of $\mathcal{F}^{\mu \nu}$ and $\tilde{\mathcal{F}}^{\mu \nu}$ as

$$
\partial_{\mu} \tilde{\mathcal{F}}^{\mu \nu}=0
$$

and

$$
\partial_{\mu} \mathcal{F}^{\mu \nu}=j^{\nu}
$$

for the homogeneous and inhomogeneous equations, respectively. 


\section{The Abelian Model U(1) and Electromagnetism}

The first example that we will consider is a complex scalar field theory. This model serves as an introduction to the mathematical methods and concepts that lie at the foundation of gauge theory. The total symmetry group considered in this section will be space-time translations and the $U(1)$ phase transformations of the fields.

\section{1: Global U(1) Theory For a Complex Scalar Field}

Consider a one-component complex scalar field $\phi=\phi(x)$, whose dynamics are determined by the action:

$$
S=\int d^{4} x \mathcal{L}\left(\phi, \partial_{\mu} \phi\right)
$$

where the Lagrange density is given by

$$
\mathcal{L}=\frac{1}{2} \partial_{\mu} \phi^{\dagger} \partial^{\mu} \phi-\frac{1}{2} \mu^{2}|\phi|^{2}-\frac{1}{4} \lambda|\phi|^{4}
$$

where $|\phi|^{2}=\phi^{\dagger} \phi$ and $\mu^{2}$ and $\lambda$ are real constants with $\lambda>0$. From the Euler-Lagrange equations (1.9)

$$
\partial_{\mu} \frac{\partial \mathcal{L}}{\partial\left(\partial_{\mu} \phi_{j}\right)}-\frac{\partial \mathcal{L}}{\partial \phi_{j}}=0
$$

we obtain the field equations for $\phi$ :

$$
\partial_{\mu} \partial^{\mu} \phi+\left(\mu^{2}+\lambda|\phi|^{2}\right) \phi=0
$$

The complex conjugate field $\phi^{\dagger}$ satisfies the corresponding equation :

$$
\partial_{\mu} \partial^{\mu} \phi^{\dagger}+\left(\mu^{2}+\lambda|\phi|^{2}\right) \phi^{\dagger}=0
$$

The action (2.1)-(2.2) is invariant under the $U(1)$ transformations which changes the phase of field and its conjugate according to

$$
\phi(x) \longrightarrow e^{i \theta} \phi(x), \quad \phi^{\dagger}(x) \longrightarrow e^{-i \theta} \phi^{\dagger}(x), \quad \forall x \in \mathbb{M}
$$


while leaving the space-time coordinates unaffected. We shall refer to these phase transformations as global because they are the same at every point $x \in \mathbb{M}$. The infinitesimal transformations $(\theta=\varepsilon)$ take the form:

$$
\begin{aligned}
x^{\mu} & \longrightarrow x^{\mu} \\
\phi(x) & \longrightarrow \phi(x)+i \phi(x) \varepsilon+\ldots \\
\phi^{\dagger}(x) & \longrightarrow \phi^{\dagger}(x)-i \phi^{\dagger}(x) \varepsilon+\ldots
\end{aligned}
$$

Using the notation of (1.12), we see that the first order changes in the coordinates and fields are

$$
\Lambda=0, \quad \Omega=i \phi(x), \quad \Omega^{\dagger}=-i \phi^{\dagger}(x)
$$

The group $U(1)$ is one dimensional, so there is only one conserved current

$$
J^{\mu}=-\frac{\partial \mathcal{L}}{\partial\left(\partial_{\mu} \phi\right)}(i \phi(x))-\frac{\partial \mathcal{L}}{\partial\left(\partial_{\mu} \phi^{\dagger}\right)}\left(-i \phi^{\dagger}(x)\right)=-i\left(\phi \partial^{\mu} \phi^{\dagger}+\phi^{\dagger} \partial^{\mu} \phi\right) .
$$

We verify the conservation equation :

$$
\begin{aligned}
\partial_{\mu} J^{\mu} & =-i \partial_{\mu}\left(\phi \partial^{\mu} \phi^{\dagger}-\phi^{\dagger} \partial^{\mu} \phi\right)=-i\left(\phi \partial_{\mu} \partial^{\mu} \phi^{\dagger}-\phi^{\dagger} \partial_{\mu} \partial^{\mu} \phi\right) \\
& =i \phi\left(\mu^{2}+\lambda|\phi|^{2}\right) \phi^{\dagger}-i \phi^{\dagger}\left(\mu^{2}+\lambda|\phi|^{2}\right) \phi=0 .
\end{aligned}
$$

The conserved charge is

$$
Q=\int d^{3} \mathbf{x} J^{0}(x)=i \int d^{3} \mathbf{x}\left(\phi^{\dagger} \partial^{0} \phi-\phi \partial^{0} \phi^{\dagger}\right) .
$$

The integrand is the charge density of the field and $Q$ is the total charge possessed by $\phi$.

The action (2.1)-(2.2) is also invariant under the four parameter group of spacetime translations:

$$
\begin{aligned}
x^{\mu} & \longrightarrow x^{\mu}+\delta_{\alpha}^{\mu} \varepsilon^{\alpha}, \quad(\operatorname{sum} \alpha=0,1,2,3) \\
\phi(x) & \longrightarrow \phi(x), \\
\phi^{\dagger}(x) & \longrightarrow \phi^{\dagger}(x),
\end{aligned}
$$


so the first order change factors are

$$
\Lambda_{\alpha}^{\mu}=\delta_{\alpha}^{\mu}, \quad \Omega=\Omega^{\dagger}=0
$$

The resulting conserved currents:

$$
J_{\alpha}^{\mu} \equiv T_{\alpha}^{\mu}=\frac{\partial \mathcal{L}}{\partial\left(\partial_{\mu} \phi\right)} \partial_{\nu} \phi \delta_{\alpha}^{\nu}+\frac{\partial \mathcal{L}}{\partial\left(\partial_{\mu} \phi^{\dagger}\right)} \partial_{\nu} \phi^{\dagger} \delta_{\alpha}^{\nu}
$$

when combined, form the energy-momentum tensor :

$$
T_{\mu \alpha}=\frac{1}{2}\left(\partial_{\mu} \phi^{\dagger} \partial_{\alpha} \phi+\partial_{\mu} \phi \partial_{\alpha} \phi^{\dagger}\right)-\mathcal{L} \delta_{\mu \alpha}
$$

the corresponding conserved charges constitute the momentum four-vector:

$$
P_{\alpha}=\int d^{3} x\left\{\frac{1}{2} \partial_{0} \phi^{\dagger} \partial_{\alpha} \phi+\frac{1}{2} \partial_{0} \phi \partial_{\alpha} \phi^{\dagger}-\mathcal{L} \delta_{0 \alpha}\right\}
$$

The components of the conserved three-momentum are

$$
P_{k}=\int d^{3} x\left\{\frac{1}{2} \partial_{0} \phi^{\dagger} \partial_{k} \phi+\frac{1}{2} \partial_{0} \phi \partial_{k} \phi^{\dagger}\right\}, \quad k=1,2,3
$$

The total conserved energy of the field is

$$
E=P_{0}=\int d^{3} \mathbf{x}\left\{\frac{1}{2} \partial_{0} \phi \partial_{0} \phi^{\dagger}+\frac{1}{2} \sum_{k=1}^{3} \partial_{k} \phi \partial_{k} \phi^{\dagger}+\frac{1}{2} \mu^{2}|\phi|^{2}+\frac{1}{4} \lambda|\phi|^{4}\right\} .
$$

Since the terms in this integral are all positive, we see that the total energy is minimized precisely when $\phi=0$. This constitutes the vacuum configuration for the globally symmetric system given by $(2.1)$. We will return to this point latter. The angular momentum of the field is also conserved due to the invariance of the action under the group of spatial rotations. But this invariance will have no relevance to our study and will not be treated here. 


\section{2: Local U(1) Symmetry, Gauge Fields}

When we treat a complex-valued field $\phi=\phi(x)$ defined all over space-time, as we have done in the previous section, there is the underlying assumption that at each point $x$, there is known an orthogonal pair of directions, which we shall call a local frame, that serves to define the real and imaginary parts of $\phi(x)$ and these local frames somehow agree everywhere in M. But the field $\phi$ is just a mathematical construct and there is no physical way of comparing frames at two different points, say, $x$ and $y$. Indeed, the special theory of relativity even asserts that there can be no communication between $x$ and $y$ if these points have a space-like separation.

We can bring these questions into clearer focus, if we consider the possibility of making local phase transformations that vary from point to point:

$$
\phi(x) \rightarrow \tilde{\phi}(x)=U(x) \phi(x)=e^{i q \alpha(x)} \phi(x)
$$

The action (2.1) fails to be invariant under local (gauge) $U(1)$ transformations. Indeed, the derivative terms $\partial_{\mu} \phi$ destroy the gauge symmetry of (2.1), as can be seen by inserting the transformed field into this derivative:

$$
\begin{aligned}
\partial_{\mu}(U(x) \phi(x)) & =\partial_{\mu}\left(e^{i q \alpha(x)} \phi(x)\right) \\
& =e^{i q \alpha(x)}\left(i q \partial_{\mu} \alpha(x)\right) \phi(x)+e^{i q \alpha(x)} \partial_{\mu} \phi(x) \\
& =U(x)\left[\partial_{\mu} \phi(x)+\left(i q \partial_{\mu} \alpha(x)\right) \phi(x)\right]
\end{aligned}
$$

Substituting this expression into (2.1) we find additional terms involving $\partial_{\mu} \alpha$ that violate invariance. To eliminate these anomalous terms, we replace the partial derivatives by covariant derivatives :

$$
\partial_{\mu} \phi(x) \rightarrow D_{\mu} \phi(x)=\partial_{\mu} \phi(x)+i q A_{\mu}(x) \phi(x),
$$

$$
\partial_{\mu} \phi^{\dagger}(x) \rightarrow D_{\mu} \phi^{\dagger}(x)=\partial_{\mu} \phi^{\dagger}(x)-i q A_{\mu}(x) \phi^{\dagger}(x),
$$

which involves the introduction of a real vector field: $A_{\mu}=A_{\mu}(x)$, called the gauge field associated with the local $U(1)$ symmetry. The coupling constant q determines 
the strength of the interaction that is analogous to the classical charge. We require that under the local $\mathrm{U}(1)$ phase transformation these gauge fields transform:

$$
A_{\mu}(x) \longrightarrow \tilde{A}_{\mu}(x)=U A_{\mu} U^{-1}-\frac{i}{q} U \partial_{\mu} U^{-1}=A_{\mu}(x)-\partial_{\mu} \alpha(x)
$$

then

$$
\begin{aligned}
\tilde{D}_{\mu} \tilde{\phi}(x) & =\partial_{\mu} \tilde{\phi}(x)+i q \tilde{A}_{\mu}(x) \tilde{\phi}(x) \\
& =\partial_{\mu}\left(e^{i q \alpha} \phi(x)\right)+i q\left(A_{\mu}(x)-\partial_{\mu} \alpha\right) e^{i q \alpha} \phi(x) \\
& =e^{i q \alpha}\left(\partial_{\mu} \phi(x)+i A_{\mu}(x) \phi(x)\right) \\
& =e^{i q \alpha} D_{\mu} \phi(x) .
\end{aligned}
$$

Similarly,

$$
\tilde{D}_{\mu} \tilde{\phi}^{\dagger}(x)=e^{-i q a} D_{\mu} \phi^{\dagger}(x) .
$$

It is clear from the transformation behavior of the covariant derivatives (2.24) that if we replace in Lagrange density (2.2) ordinary differentiation by covariant differentiation, we shall obtain an expression that is invariant under both global and local $\mathrm{U}(1)$ phase transformations.

The quantity iq $A_{\mu}(x) d x^{\mu}$ specifies the infinitesimal rotation in the frame in going from the point $x$ to the point $x+d x$. It appears in the covariant derivative to include the change in the field $\phi$ due to the rotation of this local frame and thus takes into account the total change in $\phi$. Indeed, if $\left(D_{\mu} \phi\right) d x^{\mu}=0$, this means that the change in the field components: $\partial_{\mu} \phi(x)=-i q A_{\mu}(x) \phi$ is entirely due to the rotation of the local frame.

Before attempting to modify the action (2.1)-(2.2), by introducing the covariant derivative, we must specify the gauge field $A_{\mu}$ beyond than just giving its transformation properties. To accomplish this, we introduce the curvature tensor associated 
with this field:

$$
F_{\mu \nu} \equiv \frac{-i}{q}\left[D_{\mu}, D_{\nu}\right]=\partial_{\mu} A_{\nu}-\partial_{\nu} A_{\mu}
$$

which is invariant under this the gauge transformation (2.22). The curvature 2-form is

$$
F=F_{\mu \nu} d x^{\mu} \wedge d x^{\nu}
$$

and its Hodge dual is

$$
{ }^{*} F={ }^{*} F_{\rho \sigma} d x^{\rho} \wedge d x^{\sigma}
$$

where

$$
{ }^{*} F_{\rho \sigma} \equiv \frac{1}{2} \varepsilon_{\rho \sigma \alpha \beta} F^{\alpha \beta}
$$

The four-space integral

$$
\begin{aligned}
\int F \wedge^{*} F & =\int F_{\mu \nu}{ }^{*} F_{\rho \sigma} d x^{\mu} \wedge d x^{\nu} \wedge d x^{\rho} \wedge d x^{\sigma} \\
& =\int F_{\mu \nu} \frac{1}{2} \varepsilon_{\rho \sigma \alpha \beta} F^{\alpha \beta} \varepsilon^{\mu \nu \rho \sigma} d x^{0} \wedge d x^{1} \wedge d x^{2} \wedge d x^{3} \\
& =\frac{1}{12} \int F_{\mu \nu} F^{\alpha \beta}\left(\delta_{\alpha}^{\mu} \delta_{\beta}^{\nu}-\delta_{\beta}^{\mu} \delta_{\alpha}^{\nu}\right) d x^{0} \wedge d x^{1} \wedge d x^{2} \wedge d x^{3} \\
& =\frac{1}{6} \int F_{\mu \nu} F^{\mu \nu} d^{4} x
\end{aligned}
$$

is clearly invariant under local phase transformations.

To modify the action (2.1)-(2.2) to make it invariant under local gauge transformations, we replace the ordinary derivatives of the $\phi$-fields by covariant derivatives and append a term proportional to (2.29). Our modified action is :

$$
S=\int d^{4} x \mathcal{L}\left(\phi, \partial_{\mu} \phi ; A, \partial_{\rho} A\right)
$$

where the Lagrange density is given by

$$
\mathcal{L}=\frac{1}{2} D_{\mu} \phi^{\dagger} D^{\mu} \phi-V\left(|\phi|^{2}\right)-\frac{1}{4} F_{\mu \nu} F^{\mu \nu}
$$


where the potential function is given by

$$
V\left(|\phi|^{2}\right) \equiv \frac{1}{2} \mu^{2}|\phi|^{2}+\frac{1}{4} \lambda|\phi|^{4}
$$

With the introduction of the $A$-field, the dynamics of the $\phi$-field is changed. We calculate the field equations:

$$
\begin{aligned}
0 & =\partial_{\mu} \frac{\partial \mathcal{L}}{\partial\left(\partial_{\mu} \phi_{j}^{\dagger}\right)}-\frac{\partial \mathcal{L}}{\partial \phi_{j}^{\dagger}}, \\
& =\frac{1}{2} \partial_{\mu} D^{\mu} \phi+i q A_{\mu}(x) \phi(x)-\frac{\partial V}{\partial \phi_{j}^{\dagger}},
\end{aligned}
$$

which simplifies to

$$
D_{\mu} D^{\mu} \phi+\left(\mu^{2}+\lambda|\phi|^{2}\right) \phi=0 .
$$

The complex conjugate field $\phi^{\dagger}$ satisfies the corresponding equation :

$$
D_{\mu} \cdot D^{\mu} \phi^{\dagger}+\left(\mu^{2}+\lambda|\phi|^{2}\right) \phi^{\dagger}=0 .
$$

There are a set of four Euler-Lagrange equations for the $A$-field components:

$$
\begin{aligned}
0 & =\partial_{\alpha} \frac{\partial \mathcal{L}}{\partial\left(\partial_{\alpha} A_{\beta}\right)}-\frac{\partial \mathcal{L}}{\partial A_{\beta}}, \quad(\beta=0,1,2,3) \\
& =-\partial_{\alpha} F^{\alpha \beta}-\frac{i q}{2}\left(\phi D^{\beta} \phi^{\dagger}-\phi^{\dagger} D^{\beta} \phi\right) .
\end{aligned}
$$

Next let us calculate the conserved current associated with the invariance of the modified action (2.30)-(2.31) under local $\mathrm{U}(1)$ gauge transformations. Refering to equation (1.14), we have

$$
J^{\beta}=-\frac{\partial \mathcal{L}}{\partial\left(\partial_{\beta} \phi\right)}(i q \phi(x))-\frac{\partial \mathcal{L}}{\partial\left(\partial_{\beta} \phi^{\dagger}\right)}\left(-i q \phi^{\dagger}(x)\right)=\frac{i q}{2}\left(\phi^{\dagger} D^{\beta} \phi-\phi D^{\beta} \phi^{\dagger}\right) .
$$

Thus, the field equations (2.36) for the $A$-field can be written

$$
\partial_{\alpha} F^{\alpha \beta}=J^{\beta},
$$

where

$$
J^{\beta}=\frac{i q}{2}\left(\phi^{\dagger} D^{\beta} \phi-\phi D^{\beta} \phi^{\dagger}\right)
$$


is the conserved current of the $\mathrm{U}(1)$ symmetry of the action. The corresponding conserved charge is

$$
Q=\int d^{3} \mathbf{x} J^{0}(x)=\frac{i q}{2} \int d^{3} \mathbf{x}\left(\phi^{\dagger} D^{0} \phi-\phi D^{0} \phi^{\dagger}\right)
$$

The equations (2.34), (2.35), (2.39) and (2.40) are recognized to be the analogues of (2.4), (2.5), (2.10) and (2.11) with the replacement $\partial_{\mu} \rightarrow D_{\mu}$.

Equation (2.38) constitutes four of the eight Maxwell's equations, the other four:

$$
\partial_{\mu}^{*} F^{\mu \nu}=0
$$

are the integrability conditions, which are satisfied by the curvature tensor (2.25). Indeed, we have

$$
\partial_{\mu}{ }^{*} F^{\mu \nu} \equiv \frac{1}{2} \varepsilon^{\mu \nu \alpha \beta} \partial_{\mu} F_{\alpha \beta}=\varepsilon^{\mu \nu \alpha \beta} \partial_{\mu} \partial_{\alpha} A_{\beta}=0
$$

because the alternating form $\varepsilon$ is skew-symmetric under the interchange of $\mu$ and $\alpha$, while $\partial_{\mu} \partial_{\alpha}$ is symmetric.

We rewrite the two systems of field equations $(2.34),(2.35)$ and $(2.38)$ in an expanded form that exhibits its interacting fields content:

$$
\begin{aligned}
\square \phi+\left(\mu^{2}+\lambda|\phi|^{2}\right) \phi & =i q\left(\partial_{\mu} A^{\mu}\right) \phi-q^{2} A_{\mu} A^{\mu} \phi, \\
\square \phi^{\dagger}+\left(\mu^{2}+\lambda|\phi|^{2}\right) \phi^{\dagger} & =-i q\left(\partial_{\mu} A^{\mu}\right) \phi^{\dagger}-q^{2} A_{\mu} A^{\mu} \phi^{\dagger}, \\
\square A^{\beta}-\partial^{\beta}\left(\partial_{\alpha} A^{\alpha}\right) & =\frac{i q}{2}\left(\phi^{\dagger} \partial^{\beta} \phi-\phi \partial^{\beta} \phi^{\dagger}\right)-q^{2} A^{\beta}|\phi|^{2} .
\end{aligned}
$$

The equations (2.43) constitute a system of interacting fields, with the $\phi$ and $\phi^{\dagger}$ fields carrying electrical charges of $q$ and $-q$, respectively, and acting as sources for the electromagnetic fields. This system is determined up to a gauge transformation. For example, we can fix the gauge by requiring the Coulomb/Lorentz gauge condition: $\partial_{\mu} A^{\mu}=0$; that is, make the transformation $A \rightarrow \tilde{A}=A-\partial \theta$, with $\square \theta=\partial_{\mu} A^{\mu}$, then 
system (2.43) is stripped of its inessential terms and takes the simpler form:

$$
\begin{aligned}
& \square \phi+\left(\mu^{2}+\lambda|\phi|^{2}\right) \phi=-q^{2} A_{\mu} A^{\mu} \phi, \\
& \square \phi^{\dagger}+\left(\mu^{2}+\lambda|\phi|^{2}\right) \phi^{\dagger}=-q^{2} A_{\mu} A^{\mu} \phi^{\dagger}, \\
& \square A^{\beta}=\frac{i q}{2}\left(\phi^{\dagger} \partial^{\beta} \phi-\phi \partial^{\beta} \phi^{\dagger}\right)-q^{2} A^{\beta}|\phi|^{2}, \quad \beta=0,1,2,3,
\end{aligned}
$$

which is completely determined by its initial conditions.

As a final exercise in this section, let us examine how the energy-momentum tensor has been changed with the introduction of the local $U(1)$ symmetry and the attendant introduction of the electromagnetic gauge fields. The invariance of action (2.30)-(2.31) under space-time translations imply the first order factors $\Lambda_{\alpha}^{\mu}=$ $\delta_{\alpha}^{\mu}, \Omega=0$, so the Noether's theorem (1.14) gives for the energy-momentum tensor

$$
\begin{aligned}
T_{\beta}^{\mu} & =\frac{\partial \mathcal{L}}{\partial\left(\partial_{\mu} \phi\right)} \partial_{\nu} \phi \delta_{\beta}^{\nu}+\frac{\partial \mathcal{L}}{\partial\left(\partial_{\mu} \phi^{\dagger}\right)} \partial_{\nu} \phi^{\dagger} \delta_{\beta}^{\nu}+\frac{\partial \mathcal{L}}{\partial\left(\partial_{\mu} A^{\sigma}\right)} \partial_{\nu} A^{\sigma} \delta_{\beta}^{\nu}-\mathcal{L} \delta_{\beta}^{\mu} \\
& =\frac{1}{2}\left(D^{\mu} \phi^{\dagger}\right) \partial_{\beta} \phi+\frac{1}{2}\left(D^{\mu} \phi\right) \partial_{\beta} \phi^{\dagger}-F^{\mu}{ }_{\sigma} \partial_{\beta} A^{\sigma}-\mathcal{L} \delta_{\beta}^{\mu}
\end{aligned}
$$

For simplicity, let us choose the temporal gauge in which $A^{0} \equiv 0$, so the conserved four-momentum takes the form

$$
P_{\beta}=\int d^{3} \mathbf{x} T^{0}{ }_{\beta}(x)=\int d^{3} \mathbf{x}\left\{\frac{1}{2}\left(\partial^{0} \phi^{\dagger}\right) \partial_{\beta} \phi+\frac{1}{2}\left(\partial^{0} \phi\right) \partial_{\beta} \phi^{\dagger}-F^{0 k} \partial_{\beta} A_{k}-\mathcal{L} \delta_{\beta}^{0}\right\}
$$

where we sum repeated $k=1,2,3$. The total energy of the interacting fields is

$$
\begin{aligned}
E=P_{0} & =\int d^{3} \times\left\{\left(\partial^{0} \phi^{\dagger}\right) \partial_{0} \phi-F^{0 k} \partial_{0} A_{k}-\mathcal{L}\right\} \\
& =\int d^{3} \times\left\{\left(\partial^{0} \phi^{\dagger}\right) \partial_{0} \phi-\left(\partial^{0} A^{k}\right)\left(\partial_{0} A_{k}\right)-\frac{1}{2} D_{\mu} \phi^{\dagger} D^{\mu} \phi+V\left(|\phi|^{2}\right)+\frac{1}{4} F_{\mu \nu} F^{\mu \nu},\right\} \\
& =\int d^{3} \times\left\{\frac{1}{2}\left|\partial_{0} \phi\right|^{2}+V\left(|\phi|^{2}\right)+\frac{1}{2} \sum_{k=1}^{3}\left[\left(\partial_{0} A_{k}\right)^{2}+\left|D^{k} \phi\right|^{2}\right]+\frac{1}{4} \sum_{j=1}^{3} \sum_{k=1}^{3}\left(F_{j k}\right)^{2}\right\},
\end{aligned}
$$

which is clearly non-negative. The components of the three-momentum are

$$
P_{j}=\int d^{3} \mathbf{x}\left\{\frac{1}{2}\left(\partial^{0} \phi^{\dagger}\right) \partial_{j} \phi+\frac{1}{2}\left(\partial^{0} \phi\right) \partial_{j} \phi^{\dagger}-F^{0 k} \partial_{j} A_{k}\right\}, \quad j=1,2,3
$$




\section{3: $\mathrm{SO}(2)$ Field Theory}

An alternative formulation of the $\mathrm{U}(1)$ theory is now developed using a two component real scalar field and $\mathrm{SO}(2)$ as the internal symmetry group. The dynamical content of this theory is shown to be identical to the $\mathrm{U}(1)$ case. This is reasonable as the internal spaces are the same and the symmetry groups are isomorphic. Moreover, the analysis introduces the form of representations that is used in the $\mathrm{SO}(3)$ theory considered in the next section.

\section{The Global Version}

The Lagrangian density for a two-component real field $\phi=\left(\phi^{1}, \phi^{2}\right)$ is given by

$$
L=\frac{1}{2} \partial_{\mu} \phi^{i} \partial^{\mu} \phi^{i}-V(\phi)
$$

where $V(\phi)=1 / 2 \mu^{2}\|\phi\|^{2}+1 / 4 \lambda\|\phi\|^{4}$ and where $\|\phi\|^{2}=\left(\phi^{1}\right)^{2}+\left(\phi^{2}\right)^{2}$ is the usual Euclidean norm in $\mathbb{R}^{2}$.

The Euler-Lagrange equations (1.12) lead to the following system of equations for the $\phi$ field

$$
\square \phi^{i}+\mu^{2} \phi^{i}+\lambda\|\phi\|^{2} \phi^{i}=0, \quad i=1,2
$$

Since the Lagrange density (2.49) involves only quadratic terms in $\phi$, it is invariant under space-time independent $\mathrm{SO}(2)$ transformations of the form

$$
\phi \longrightarrow \tilde{\phi}=U(\theta) \phi
$$

where $U(\theta) \in S O(2)$. The matrix $U(\theta)$ can be expressed as

$$
U(\theta)=\left(\begin{array}{cc}
\cos (\theta) & -\sin (\theta) \\
\sin (\theta) & \cos (\theta)
\end{array}\right)
$$

This $\mathrm{SO}(2)$ element can also be described in terms of the generator

$$
\xi=i\left(\begin{array}{cc}
0 & i \\
-i & 0
\end{array}\right)
$$


by

$$
U(\theta)=\left(\begin{array}{cc}
\cos (\theta) & -\sin (\theta) \\
\sin (\theta) & \cos (\theta)
\end{array}\right)=e^{i \theta \xi}
$$

We see that the generator $\xi$ plays the same role as i in (2.6). Under an infinitesimal transformation, $|\theta|<<1$,

$$
\phi \longrightarrow \tilde{\phi}=(I+i \theta \xi) \phi
$$

the field components transform according to

$$
\begin{aligned}
& \phi^{1} \longrightarrow \tilde{\phi}^{1}=\phi^{1}-\theta \phi^{2} \\
& \phi^{2} \longrightarrow \tilde{\phi}^{2}=\phi^{2}+\theta \phi^{1}
\end{aligned}
$$

The first order transformation factors $\Omega$ take the values $\Omega^{i}{ }_{i}=0, \Omega^{1}{ }_{2}=-\phi^{2}$, and $\Omega^{2}{ }_{1}=$ $\phi^{1}$. Thus the conserved $\mathrm{SO}(2)$ currents derived from (1.16) are

$$
J^{\mu}=-\frac{\partial \mathcal{L}}{\partial\left(\partial_{\mu} \phi^{1}\right)}\left(-\phi^{2}\right)-\frac{\partial \mathcal{L}}{\partial\left(\partial_{\mu} \phi^{2}\right)}\left(\phi^{1}\right)=\phi^{2} \partial^{\mu} \phi^{1}-\phi^{1} \partial^{\mu} \phi^{2}
$$

Comparing this to (2.9) shows that the two current densities are the same. With $\phi=\phi^{1}+i \phi^{2}$, equation $(2.9)$ reads

$$
J^{\mu}=\frac{i}{2}\left\{\left(\phi^{1}-i \phi^{2}\right) \partial^{\mu}\left(\phi^{1}+i \phi^{2}\right)-\left(\phi^{1}+i \phi^{2}\right) \partial^{\mu}\left(\phi^{1}-i \phi^{2}\right)\right\}=\phi^{2} \partial^{\mu} \phi^{1}-\phi^{1} \partial^{\mu} \phi^{2}
$$

Turning to the conserved energy-momentum

$$
T^{\mu}{ }_{\nu}=\sum_{j=1}^{2} \frac{\partial \mathcal{L}}{\partial\left(\partial_{\mu} \phi^{j}\right) \partial_{\nu} \phi^{j}}-\mathcal{L} \delta^{\mu}{ }_{\nu},
$$

we find

$$
T^{\mu}{ }_{\nu}=\sum_{j=1}^{2} \partial^{\mu} \phi^{j} \partial_{\nu} \phi^{j}-\mathcal{L} \delta_{\nu}^{\mu}
$$

The total energy is identical to that calculated in (2.18):

$$
\begin{aligned}
E & =\int d^{3} x T_{0}^{0}=\int d^{3} x\left\{\partial^{0} \phi^{i} \partial_{0} \phi^{i}-\frac{1}{2} \partial^{\mu} \phi^{i} \partial_{\mu} \phi^{i}+V\left(\phi^{2}\right)\right\} \\
& =\int d^{3} x\left\{\frac{1}{2} \partial_{0} \phi^{i} \partial^{0} \phi^{i}+\frac{1}{2} \nabla \phi^{i} \cdot \nabla \phi^{i}+\frac{1}{2} \mu^{2} \phi^{2}+\frac{1}{4} \lambda \phi^{4}\right\}
\end{aligned}
$$




\section{The Local Version}

As in the $U(1)$ case, the Lagrangian density (2.49) does not possess symmetry under the local transformations:

$$
\phi(x) \longrightarrow \bar{\phi}(x)=U(x) \phi(x)
$$

where $U(x)=e^{i \theta(x) \xi}$ in terms of the generator $\xi$ of $\mathrm{SO}(2)$ given in (2.53). Again the term that destroys invariance under the gauge transformation (2.62) is the derivative operator $\partial_{\mu}$ :

$$
\begin{aligned}
{\left[\partial_{\mu}(U(x) \phi(x))\right]^{i} } & =U^{i}{ }_{j} \partial_{\mu} \phi^{j}+\left(\partial_{\mu} U^{i}{ }_{j}(x)\right) \phi^{j} \\
& =U^{i}{ }_{j} \partial_{\mu} \phi^{j}+\left(i U \xi \partial_{\mu} \theta\right)_{k}^{i} \phi^{k} \\
& =U^{i}{ }_{j} \partial_{\mu} \phi^{j}+i U_{j}^{i} \xi_{k}^{j}\left(\partial_{\mu} \theta\right) \phi^{k} \\
& =U^{i}{ }_{j}\left[\partial_{\mu} \phi^{j}+i\left(\partial_{\mu} \theta\right) \xi_{k}^{j} \phi^{k}\right]
\end{aligned}
$$

Again, we introduce the connection potential into the derivative operator that compensates for the transformation (2.62). In this case, the covariant derivative takes the form

$$
\left(D_{\mu} \phi\right)^{i}=\partial_{\mu} \phi^{i}+i q A_{\mu} \xi_{j}^{i} \phi^{j}
$$

so that

$$
\begin{aligned}
& \left(D_{\mu} \phi\right)^{1}=\partial_{\mu} \phi^{1}-q A_{\mu} \phi^{2} \\
& \left(D_{\mu} \phi\right)^{2}=\partial_{\mu} \phi^{2}+q A_{\mu} \phi^{1}
\end{aligned}
$$

for the individual fields. The locally $\mathrm{SO}(2)$-invariant Lagrange density is

$$
\mathcal{L}=\frac{1}{2}\left(D_{\mu} \phi\right)^{i}\left(D^{\mu} \phi\right)^{i}-V\left(\phi^{2}\right)+\frac{1}{4} \mathcal{F}_{\mu \nu} \mathcal{F}^{\mu \nu}
$$

where, as we will see, the field strength for the $A_{\mu}$ potential is defined as in (2.25).

Since

$$
|\phi|^{2}=\phi^{\dagger} \phi=\left(\phi^{1}-i \phi^{2}\right)\left(\phi^{1}+\phi^{2}\right)=\phi^{2}=\left(\phi^{1}\right)^{2}+\left(\phi^{2}\right)^{2},
$$


the comparison to local $\mathrm{U}(1)$ theory rests in comparing the covariant derivative terms in (2.31) and (2.66). With $\phi=\phi^{1}+i \phi^{2}$, the derivative terms in (2.31) become

$$
\begin{aligned}
\left(D^{\mu} \phi\right)^{\dagger}\left(D_{\mu} \phi\right) & =\left(\partial^{\mu} \phi^{\dagger}-i q A^{\mu} \phi^{\dagger}\right)\left(\partial_{\mu} \phi+i q A_{\mu} \phi\right) \\
& =\partial^{\mu} \phi^{\dagger} \partial_{\mu} \phi+i q A_{\mu}\left(\partial^{\mu} \phi^{\dagger} \phi-\partial^{\mu} \phi \phi^{\dagger}\right)+q^{2} A_{\mu} A^{\mu}(\phi)^{2} \\
& =\left(\partial^{\mu} \phi^{i}\right)\left(\partial_{\mu} \phi^{i}\right)+2 q A^{\mu}\left(\partial^{\mu} \phi^{2} \phi^{1}-\partial^{\mu} \phi^{1} \phi^{2}\right)+q^{2} A_{\mu} A^{\mu} \phi^{2}
\end{aligned}
$$

while

$$
\begin{aligned}
\left(D^{\mu} \phi\right)^{i}\left(D_{\mu} \phi\right)^{i} & =\left(\partial_{\mu} \phi^{1}-q A_{\mu} \phi^{2}\right)\left(\partial^{\mu} \phi^{1}-q A^{\mu} \phi^{2}\right)+\left(\partial_{\mu} \phi^{2}+q A_{\mu} \phi^{1}\right)\left(\partial^{\mu} \phi^{2}+q A^{\mu} \phi^{1}\right) \\
& =\left(\partial^{\mu} \phi^{i}\right)\left(\partial_{\mu} \phi^{i}\right)+2 q A^{\mu}\left(\partial^{\mu} \phi^{2} \phi^{1}-\partial^{\mu} \phi^{1} \phi^{2}\right)+q^{2} A_{\mu} A^{\mu} \phi^{2}
\end{aligned}
$$

from (2.66). The remaining term in (2.66) can be determined by computing the commutatoring $\left[D_{\mu}, D_{\nu}\right]$. Applying the operator $D_{\mu}$ to $D_{\nu} \phi$, we get

$$
\begin{aligned}
\left(D_{\mu}\left(D_{\nu} \phi\right)\right)^{i} & =\partial\left(D_{\nu} \phi\right)^{i}+i q A_{\mu} \xi_{j}^{i}\left(D_{\nu} \phi\right)^{j} \\
& =\partial_{\mu}\left(\partial_{\nu} \phi^{i}+i q A_{\nu} \xi_{k}^{i} \phi^{k}\right)+i q A_{\mu} \xi_{j}^{i}\left(\partial_{\nu} \phi^{j}+i q A_{\nu} \xi_{k}^{j} \phi^{k}\right) \\
& =\partial_{\mu} \partial_{\nu} \phi^{i}+i q\left[\partial_{\mu} A_{\nu} \xi_{k}^{i} \partial_{\mu} \phi^{k}+A_{\nu} \xi_{k}^{i} \partial_{\mu} \phi^{k}\right]+i q A_{\mu} \xi_{j}^{i} \partial_{\nu} \phi^{j}-q^{2} A_{\mu} A_{\nu} \xi_{j}^{i} \xi_{k}^{j} \phi^{k}
\end{aligned}
$$

On the other hand, the ith component of $D_{\nu}\left(D_{\mu} \phi\right)$ with $\mu$ exchanged with $\nu$. The difference of these two expressions, multiplied by $(i q)^{-1}$ yields the field strength tensor, the coefficients of which are

$$
\mathcal{F}_{\mu \nu}=\partial_{\mu} A_{\nu}-\partial_{\nu} A_{\mu}
$$

which is identical to (2.25). Thus, we see that the Lagrangian densities for $U(1)$ and $\mathrm{SO}(2)$ are equivalent. Consequently, the two theories have the same physical content. 


\section{SO(3) Field Theory}

The principles and techniques of gauge theory will now be extended to a field theory that includes a non-abelian symmetry group. The theory describes the dynamics of a triplet of real scalar fields with a total symmetry group consisting of the space-time translations and internal $\mathrm{SO}(3)$ rotations of the fields. As in the abelian case, the equations of motion and the Noether currents will be derived for both globally and locally invariant systems. The number of conserved charges admitted by the theory is shown to be related to the size of the symmetry group, that is, the number of generators. Furthermore, the local theory will include three vector fields that are analogous to electromagnetic vector potential encountered in the last section. The Noether currents, the form of the field strength, and the interactions associated with these gauge fields are shown to reflect the non-commutative structure of $\mathrm{SO}(3)$.

\section{Section 3.1: Global SO(3) Invariance}

The Lagrangian density for the global SO(3) theory is

$$
\mathcal{L}=\frac{1}{2}\left(\partial_{\mu} \phi^{i}\right)\left(\partial^{\mu} \phi^{i}\right)-V(\phi)
$$

where $V(\|\phi\|)=\frac{1}{2} \mu^{2}\|\phi\|^{2}+\frac{1}{4} \lambda\|\phi\|^{4}$. Notice that this Lagrangian density is identical in form to (2.49) except that $\phi=\left(\phi^{1}, \phi^{2}, \phi^{3}\right)$ contains an additional component and $\|\phi\|^{2} \equiv\left(\phi^{1}\right)^{2}+\left(\phi^{2}\right)^{2}+\left(\phi^{3}\right)^{2}$. The equations of motion that derive from (3.1) are

$$
\square \phi^{i}+\mu^{2} \phi^{i}+\lambda\|\phi\|^{2} \phi^{i}=0, \quad(i=1,2,3) .
$$

The energy-momentum tensor also retains the same form as in Section 2.3:

$$
T^{\mu}{ }_{\nu}=\left(\partial^{\mu} \phi^{i}\right)\left(\partial_{\nu} \phi^{i}\right)-\mathcal{L} \delta_{\nu}^{\mu}
$$


as does the total energy

$$
\begin{aligned}
E & =\int d^{3} x T_{0}^{0}=\int d^{3} x\left\{\partial^{0} \phi^{i} \partial_{0} \phi^{i}-\frac{1}{2} \partial^{\mu} \phi^{i} \partial_{\mu} \phi^{i}+V\left(\phi^{2}\right)\right\} \\
& =\int d^{3} x\left\{\frac{1}{2} \partial_{0} \phi^{i} \partial^{0} \phi^{i}+\frac{1}{2} \nabla \phi^{i} \cdot \nabla \phi^{i}+\frac{1}{2} \mu^{2}\|\phi\|^{2}+\frac{1}{4} \lambda\|\phi\|^{4}\right\} .
\end{aligned}
$$

The Lagrangian (3.1) is invariant under global rotations of the scalar field $\phi$ of the form

$$
\phi \rightarrow \tilde{\phi}=U\left(\theta^{a}\right) \phi
$$

where $U\left(\theta^{a}\right) \in S O(3)$ and the $\theta^{a}$ specify the angles of rotation about the different axes in $\mathbb{R}^{3}$. The conserved currents associated with this symmetry are determined by expressing the group element in (3.5) in terms of the matrix generators of $\mathrm{SO}(3)$. The standard representation for these generators is

$$
\left(\tau_{a}\right)_{j}^{i}=\frac{i}{2} \varepsilon_{a i j}
$$

and they satisfy the commutation relations

$$
\left[\tau_{a}, \tau_{b}\right]=\frac{i}{2} \epsilon_{a b c} \tau_{c}
$$

$U\left(\theta^{a}\right)$ can then be represented by

$$
U\left(\theta^{a}\right)=\exp \left\{i \sum_{a=1}^{3} \theta^{a} \tau_{a}\right\} .
$$

The infinitesimal version of (3.5), given in terms of the $\tau_{a}$, is

$$
\phi^{i} \rightarrow \tilde{\phi}^{i}=\phi^{i}+i \delta \theta^{a}\left(\tau_{a}\right)^{i}{ }_{j} \phi^{j}
$$

or

$$
\tilde{\phi}^{i}=\phi^{i}+\delta \theta^{a} \Omega_{a}^{i},
$$


where $\Omega_{a}^{i}=-\epsilon_{a i j} \phi^{j}$ are the first order transformation terms appearing in Noether's theorem. The global SO(3) currents are found from (1.13)

$$
J_{a}^{\mu}=-\sum_{j=1}^{3} \frac{\partial \mathcal{L}}{\partial\left(\partial_{\mu} \phi^{j}\right)} \Omega_{a j}=\sum_{j=1}^{3} \epsilon_{a j k}\left(\partial^{\mu} \phi^{j}\right) \phi^{k}
$$

Explicitly,

$$
\begin{aligned}
& J_{1}^{\mu}=\left(\partial^{\mu} \phi^{2}\right) \phi^{3}-\left(\partial^{\mu} \phi^{3}\right) \phi^{2}, \\
& J_{2}^{\mu}=\left(\partial^{\mu} \phi^{3}\right) \phi^{1}-\left(\partial^{\mu} \phi^{1}\right) \phi^{3}, \\
& J_{3}^{\mu}=\left(\partial^{\mu} \phi^{1}\right) \phi^{2}-\left(\partial^{\mu} \phi^{2}\right) \phi^{1} .
\end{aligned}
$$

Thus, the additional field component and the larger symmetry group result in two additional current densities than the abelian case. This expresses the fact that the conserved quantities in Noether's theorem are connected to the number of generators of the symmetry group. The charge densities $J_{1}^{0}, J_{2}^{0}, J_{3}^{0}$ in (3.12) lead to three independent charges found by integrating over the spatial dimensions:

$$
Q_{a}=\int d^{3} x\left\{J_{a}^{0}\right\}=\int d^{3} x\left\{\epsilon_{a i j}\left(\partial^{\mu} \phi^{i}\right) \phi^{j}\right\}, \quad(a=1,2,3)
$$

The presence of $\epsilon_{a i j}$ in the expression for these conserved charges indicates that they correspond to the generators of the underlying $\mathrm{SO}(3)$ symmetry [5].

\section{Section 3.2: The Local Theory}

The local generalization of (3.5) is given by the expression

$$
\phi(x) \rightarrow \tilde{\phi}(x)=U\left(\theta^{a}(x)\right) \phi(x)
$$

where the rotation angles, $\theta^{a}$, are now functions of space-time. The derivatives in the Lagrangian (3.1) again produce terms that destroy invariance under this type of transformation of the field components. Invariance is established by introducing additional fields into the derivative that compensate for this local transformation. 
Since the symmetry group possesses three independent generators, three gauge fields must be included in the theory. The interaction of the scalar and these additional fields is specified by the form of the gauge covariant derivative:

$$
\left(D_{\mu} \phi\right)^{i}=\partial_{\mu} \phi^{i}+i g A_{\mu}^{a}\left(\tau_{a}\right)^{i}{ }_{j} \phi^{j}
$$

where $\mathrm{g}$ is a coupling constant. Furthermore, each of the $A$ fields gives rise to a field strength tensor that is found by evaluating the commutator $\left[D_{\mu}, D_{\nu}\right]$ :

$$
\mathcal{F}_{\mu \nu}^{a}=\partial_{\mu} A_{\nu}^{a}-\partial_{\nu} A_{\mu}^{a}+i g\left(\tau_{a}\right)_{b c} A_{\mu}^{b} A_{\nu}^{c}
$$

The quadratic terms in the $A^{a}$ are consequences of the fact that the generators of $\mathrm{SO}(3)$ do not commute and imply that the interaction of these fields is non-linear. The gauge invariant Lagrangian is then

$$
\mathcal{L}=\frac{1}{2}\left(D_{\mu} \phi\right)^{i}\left(D^{\mu} \phi\right)^{i}-V(\phi)-\frac{1}{4} \mathcal{F}_{\mu \nu}^{a} \mathcal{F}_{a}^{\mu \nu}
$$

The Euler-Lagrange equations (1.9) imply the following system for the $\phi$ fields

$$
\partial_{\mu}\left(D^{\mu} \phi\right)^{i}+i g\left(\tau^{i}\right)_{j k} A_{\mu}^{k}\left(D^{\mu} \phi\right)^{j}+\mu^{2} \phi^{i} \lambda\|\phi\|^{2} \phi^{i}=0
$$

or, equivalently,

$$
\left(D_{\mu} D^{\mu} \phi\right)^{i}+\mu^{2} \phi^{i}+\lambda\|\phi\|^{2} \phi^{i}=0, \quad(i=1,2,3)
$$

Also we have

$$
\partial_{\mu} \frac{\partial \mathcal{L}}{\partial\left(\partial_{\mu} A_{\nu}^{k}\right)}-\frac{\partial \mathcal{L}}{\partial\left(A_{\nu}^{k}\right)}=0, \quad(k=1,2,3 \quad \text { and } \quad \nu=0,1,2,3)
$$

for the $A$ fields, or, explicitly,

$$
\partial_{\mu}\left(-\mathcal{F}_{k}^{\mu \nu}\right)-i g\left(\tau_{k}\right)_{j i} \phi^{i}\left(D^{\nu} \phi\right)^{j}-\frac{1}{2} i g\left(\tau_{a}\right)_{b c}\left(\delta_{b}^{k} \delta_{\alpha}^{\nu} A_{\beta}^{c}+\delta_{c}^{k} \delta_{\beta}^{\nu} A_{\alpha}^{b}\right) \mathcal{F}_{a}^{\alpha \beta}=0
$$


that is,

$$
\partial_{\mu}\left(\mathcal{F}_{k}^{\mu \nu}\right)+i g\left(\tau_{k}\right)_{i j}\left(\left(D^{\nu} \phi\right)^{i} \phi^{j}+\mathcal{F}_{i}^{\mu \nu} A_{\mu}^{j}\right)=0
$$

The additional terms in (3.17) generate an energy-momentum tensor of the form

$$
T_{\nu}^{\mu}=\frac{\partial \mathcal{L}}{\partial\left(\partial_{\mu} \phi^{i}\right)} \partial_{\nu} \phi^{i}+\frac{\partial \mathcal{L}}{\partial\left(\partial_{\mu} A_{\alpha}^{k}\right)} \partial_{\nu} A_{\alpha}^{k}-\mathcal{L} \delta_{\nu}^{\mu}
$$

which, in our case, gives

$$
T^{\mu}{ }_{\nu}=\left(D^{\mu} \phi\right)^{i} \partial_{\nu} \phi^{i}-\mathcal{F}_{k}^{\mu \alpha} \partial_{\nu} A_{\alpha}^{k}-\mathcal{L} \delta_{\nu}^{\mu}
$$

We can simplify the expression for the total energy of the system by first choosing the temporal gauge in which the time components of the A fields vanish: $A^{a}{ }_{0}=0$. Under this gauge choice, the following hold

$$
\begin{aligned}
\left(D^{0} \phi\right)^{i} & =\partial^{0} \phi^{i} \\
\mathcal{F}_{k}^{0 \alpha} \partial_{0} A_{\alpha}^{k} & =\mathcal{F}_{k}^{0 j} \partial_{0} A_{j}^{k}=\left(\partial^{0} A_{k}^{j}\right)\left(\partial_{0} A_{j}^{k}\right) \\
\mathcal{F}_{\mu \nu}^{k} \mathcal{F}_{k}^{\mu \alpha} & =\frac{1}{2}\left(\partial_{0} A_{j}^{k}\right)\left(\partial^{0} A_{k}^{j}\right)+\frac{1}{4} \mathcal{F}_{i j}^{k} \mathcal{F}_{k}^{i j}
\end{aligned}
$$

Thus, the total energy is

$$
E=\int d^{3} x\left\{\frac{1}{2}\left(\partial_{0} \phi^{i}\right)^{2}+\frac{1}{2}\left(D_{j} \phi\right)^{i}\left(D^{j} \phi\right)^{i}+\mu^{2} \phi^{2}+\frac{1}{4} \lambda \phi^{4}+\frac{3}{2}\left(\partial_{0} A_{j}^{k}\right)\left(\partial^{0} A_{k}^{j}\right)+\frac{1}{4} \mathcal{F}_{i j}^{k} \mathcal{F}_{k}^{i j}\right\}
$$

Again we see that, under the assumption $\mu^{2}>0$, the minimum energy configuration for the system is that for which $\phi=0$ and $A^{a}=0$, with $\mathrm{a}=1,2,3$.

Next we calculate the conserved currents that result from the local $\mathrm{SO}(3)$ symmetry. The infinitesimal form of the $\mathrm{SO}(3)$ transformation on the variables that make up the Lagrangian (3.17) is

$$
\begin{gathered}
x \longrightarrow x \\
\phi_{j}(x) \longrightarrow \phi_{j}(x)+i g\left(\tau_{a}\right)_{j k} \phi^{k}(x) \delta \theta^{a} \\
A_{\nu}^{j}(x) \longrightarrow A_{\nu}^{j}(x)+i g\left(\tau_{a}\right)_{k} \phi^{k}(x) \delta \theta^{a} .
\end{gathered}
$$


The three conserved currents are

$$
\begin{aligned}
J_{a}^{\mu} & =-\frac{\partial \mathcal{L}}{\partial\left(\partial_{\mu} \phi_{j}\right)}\left(i g\left(\tau_{a}\right)_{j k} \phi^{k}\right)-\frac{\partial \mathcal{L}}{\partial\left(\partial_{\mu} A_{\nu}^{b}\right)}\left(i g\left(\tau_{a}\right)_{c}^{b} A_{\nu}^{c}\right) \\
& =-i g\left(\tau_{a}\right)_{i j}\left(\left(D^{\nu} \phi\right)^{i} \phi^{j}+\mathcal{F}_{i}^{\mu \nu} A_{\mu}^{j}\right)
\end{aligned}
$$

Unlike the $U(1)$ case (2.39), the local SO(3) current densities in (3.28) have additional terms involving the gauge fields. This is a consequence of the non-abelian structure of the symmetry group and the interaction of the vector fields. On the other hand, the $\mathrm{SO}(3)$ currents that are obtained from the symmetry principle are consistent with the previous results: the $J_{a}^{\mu}$ serve as source terms for the field strength, just as in (2.38) for the U(1) theory. Furthermore, the charges derived from (3.28) exhibit the same structure and interpretation as in (3.13). In the temporal gauge, the time components of the current four-vectors reduce to

$$
J_{a}^{0}=-i g\left(\tau_{a}\right)_{i j}\left(\left(D^{0} \phi\right)^{i} \phi^{j}+\left(\partial^{0} A_{i}^{\nu}\right) A_{\nu}^{j}\right)
$$

from which the charges are computed by integration:

$$
Q_{a}=\int d^{3} x\left\{-i g\left(\tau_{a}\right)_{i j}\left(\left(D^{0} \phi\right)^{i} \phi^{j}+\left(\partial^{0} A_{i}^{\nu}\right) A_{\nu}^{j}\right)\right\}
$$




\section{Symmetry Breaking}

Spontaneous symmetry breaking occurs in a Lagrangian field theory when the theory admits states of lowest energy in which the system will no longer exhibit the full symmetry possessed by its action functional. Perturbation from the lowest energy states will reveal a particle content that is not apparent in the original Lagrangian. The effects of symmetry breaking will be shown to depend on the type of symmetry in the theory, that is, whether the initial symmetry is global or local. In this section, we will consider this concept by breaking the $\mathrm{SO}(3)$ symmetry just analyzed, by taking the parameter $\mu^{2}$ to be negative. Though, under this condition, $\mu$ can no longer be regarded as a mass, the following analysis will show that it is responsible for masses that appear in the resulting theory.

\section{Section 4.1 SO(3) Symmetry Breaking}

Recall that the globally invariant Lagrangian density examined in Section 3 is

$$
\mathcal{L}=\frac{1}{2}\left(\partial_{\mu} \phi^{i}\right)\left(\partial^{\mu} \phi^{i}\right)-V(\phi)
$$

where the potential function is

$$
V(\phi)=\frac{1}{2} \mu^{2}\|\phi\|^{2}+\frac{1}{4} \lambda\|\phi\|^{4}
$$

We remarked that, under the assumption that $\mu^{2}>0$, the energy is minimized precisely when $\phi \equiv 0$. In this section we are interested in the case where $\mu^{2}<0$, then the total energy

$$
E=\int d^{3} x\left\{\frac{1}{2}\left(\partial_{0} \phi^{i}\right)^{2}+\frac{1}{2} \sum_{j=1}^{3}\left(\partial_{j} \phi^{i}\right)\left(\partial^{j} \phi^{i}\right)+\mu^{2}\|\phi\|^{2}+\frac{1}{4} \lambda\|\phi\|^{4}\right\}
$$

is an extremum when $\partial_{\mu} \phi^{i}=0$ and $\frac{\partial V}{\partial \phi^{i}}=0$. The second condition reads

$$
\mu^{2} \phi^{i}+\lambda\|\phi\|^{2} \phi^{i}=0
$$


from which we see that either $\phi^{i}=0$, which gives zero potential energy, or

$$
\|\phi\|^{2}=\frac{-\mu^{2}}{\lambda} \equiv v^{2}
$$

which gives a still lower potential energy, namely $V=-(\lambda / 4) v^{4}$. There will be some simplification and no affect on the physics, if we reset the energy scale by adding the constant $(\lambda / 4) v^{4}$ to the potential energy. Thus, we redefine for the remainder of this paper:

$$
V(\phi)=\frac{1}{4} \lambda\left(v^{2}-\|\phi\|^{2}\right)^{2}
$$

This will ensure that, when included in the energy (4.3), fields satisfying (4.5) will contribute no potential energy to this integral.

In contrast to the result in Section 3, the lowest energy state for the Lagrangian (4.1) with $\mu^{2}<0$ is degenerate; any constant field configuration satisfying (4.5) minimizes the total energy. A generic choice for such a field configuration is

$$
\phi_{0}=\left(\begin{array}{l}
0 \\
0 \\
v
\end{array}\right) .
$$

When we expand the Lagrangian density in terms of a perturbation field $\psi(x)=$ $\phi(x)-\phi_{o}$, using the potential defined in (4.6), then

$$
\mathcal{L}=\frac{1}{2} \partial_{\mu} \psi^{i} \partial^{\mu} \psi^{i}-V\left(\psi+\phi_{0}\right)
$$

Setting

$$
\tilde{\psi}=\left(\begin{array}{l}
\psi^{1} \\
\psi^{2}
\end{array}\right)
$$

the potential term becomes

$$
\begin{aligned}
V\left(\psi+\phi_{0}\right) & =\frac{1}{4} \lambda\left(\left\|\psi+\phi_{0}\right\|^{2}-v^{2}\right)^{2} \\
& =\frac{1}{4} \lambda\left(\|\tilde{\psi}\|^{2}+\left(\psi^{3}\right)^{2}+2 v \psi^{3}\right)^{2} \\
& =\frac{1}{4} \lambda\left(\|\tilde{\psi}\|^{4}+2\|\tilde{\psi}\|^{2}\left(\left(\psi^{3}\right)^{2}+2 v \psi^{3}\right)+\left(\left(\psi^{3}\right)^{2}+2 v \psi^{3}\right)^{2}\right) \\
& =\frac{1}{4} \lambda\|\tilde{\psi}\|^{4}+\frac{1}{2} \lambda\|\tilde{\psi}\|^{2}\left(\psi^{3}\right)^{2}+\lambda v\|\tilde{\psi}\|^{2} \psi^{3}+\frac{1}{4} \lambda\left(\psi^{3}\right)^{4}+\lambda v\left(\psi^{3}\right)^{3}+\lambda v^{2}\left(\psi^{3}\right)^{2}
\end{aligned}
$$


where $\|\tilde{\psi}\|^{2} \equiv\left(\psi^{1}\right)^{2}+\left(\psi^{2}\right)^{2}$. Thus, the potential has the form

$$
V\left(\psi+\phi_{0}\right)=\frac{1}{4} \lambda\|\tilde{\psi}\|^{4}+\frac{1}{2} \lambda\|\tilde{\psi}\|^{2}\left(\psi^{3}\right)^{2}+\lambda v\|\tilde{\psi}\|^{2} \psi^{3}+\frac{1}{4} \lambda\left(\psi^{3}\right)^{4}+\lambda v\left(\psi^{3}\right)^{3}-\mu^{2}\left(\psi^{3}\right)^{2}
$$

This last expression shows that with the choice of $\phi_{0}$ as our ground configuration the full $\mathrm{SO}(3)$ symmetry is no longer manifest, but that (4.8) will still contain a recognizable $\mathrm{SO}(2)$ symmetry about the $\psi^{3}$ axis. In addition, (4.11) no longer contains mass terms in the $\psi^{1}$ and $\psi^{2}$ fields, and that the $\psi^{3}$ field has a mass term of $-\mu^{2}$, which is positive. This agrees with the Goldstone theorem [3]: the two massless boson fields that appear in the theory correspond to the two broken generators of

$\mathrm{SO}(3)$ and the remaining field acquires a physical mass $\sqrt{-2 \mu^{2}}$ as can be seen from of the Euler-Lagrange equations

$$
\begin{aligned}
\partial_{\mu} \partial^{\mu} \psi^{3}+2 \mu^{2} \psi^{3}-\lambda\|\tilde{\psi}\|^{2}\left(\psi^{3}+v\right)-\lambda\left(\psi^{3}\right)^{2}\left(\psi^{3}+v\right) & =0, \\
\partial_{\mu} \partial^{\mu} \psi^{i}-\lambda\|\tilde{\psi}\|^{2} \psi^{i}-\lambda\left(\psi^{3}\right)^{2} \psi^{i}-\left(2 \lambda v \psi^{3}\right) \psi^{i} & =0, \quad i=1,2 .
\end{aligned}
$$

\section{Section 4.2 Local SO(3) Symmetry Breaking, the Higgs Mechanism}

Now we will consider the effects of breaking the local gauge symmetry of the Lagrangian (3.17) with the condition that $\mu^{2}<0$. The approach is the same to that previously considered: seek configurations that minimize the energy of the system and expand about a particular choice of vacuum state. We shall see that one of the scalar fields retains the physical mass acquired through the expansion in Section 4.1. Differences resulting from the gauge freedom in (3.17) will include the appearance of two massive vector fields in favor of the massless scalar fields derived above [3].

The Lagrangian density associated with the locally SO(3) invariant field theory is

$$
\mathcal{L}=\frac{1}{2}\left(D_{\mu} \phi\right)^{i}\left(D^{\mu} \phi\right)^{i}-V\left(\|\phi\|^{2}\right)-\frac{1}{4} \mathcal{F}_{\mu \nu}^{a} \mathcal{F}_{a}^{\mu \nu}
$$


For $\mu^{2}<0$, the energy $(3.26)$ is minimized when $\|\phi\|^{2}=v^{2}, \partial_{\mu} \phi^{i}=0$, and $A^{a}=0$ for all $\mathrm{i}$ and a. Choosing the lowest energy state to be

$$
\phi_{0}=\left(\begin{array}{l}
0 \\
0 \\
v
\end{array}\right),
$$

any perturbation about this configuration has the form

$$
\phi=\left(\begin{array}{c}
\phi^{1} \\
\phi^{2} \\
\eta+v
\end{array}\right) .
$$

In addition, we include $A$ fields which are the perturbations about their zero values.

To determine how $\mathcal{L}$ appears with this choice of expansion field, we first choose a gauge that aligns $\phi$ with the third axis of $\mathbb{R}^{3}$. By redefining $\eta$ as necessary, the field now takes the form

$$
\phi=\left(\begin{array}{c}
0 \\
0 \\
\eta+v
\end{array}\right)
$$

In this gauge, the derivative terms in (4.13) become

$$
\begin{aligned}
& D_{\mu} \phi^{1}=\partial_{\mu} \phi^{1}+i g A_{\mu}^{a}\left(\tau_{a}\right)_{j}^{1} \phi^{j}=\frac{g}{2} A_{\mu}^{2}(\eta+v) \\
& D_{\mu} \phi^{2}=\partial_{\mu} \phi^{2}+i g A_{\mu}^{a}\left(\tau_{a}\right)_{j}^{2} \phi^{j}=-\frac{g}{2} A_{\mu}^{1}(\eta+v) \\
& D_{\mu} \phi^{3}=\partial_{\mu} \phi^{3}+i g A_{\mu}^{a}\left(\tau_{a}\right)_{j}^{3} \phi^{j}=\partial_{\mu} \eta,
\end{aligned}
$$

while the potential term in (4.13) is

$$
V(\phi)=V(\eta+v)=\frac{1}{4} \lambda\left((\eta+v)^{2}-v^{2}\right)^{2} .
$$

Expanding, we obtain

$$
\begin{aligned}
V(\eta+v) & =\frac{1}{4} \lambda\left((\eta+v)^{2}-v^{2}\right)^{2} \\
& =\frac{1}{4} \lambda\left(\eta^{2}+2 v \eta\right)^{2} \\
& =\frac{1}{4} \eta^{4}+\lambda v \eta^{3}+\lambda v^{2} \eta^{2} .
\end{aligned}
$$

Thus, with this choice of gauge, the density (4.13) is

$$
\mathcal{L}=\frac{1}{2} \partial_{\mu} \eta \partial^{\mu} \eta-\frac{1}{2}\left(\frac{g^{2}}{4}\right)\left(A_{\mu}^{1} A_{1}^{\mu}+A_{\mu}^{2} A_{2}^{\mu}\right)(\eta+v)^{2}+\mu^{2} \eta^{2}-\frac{1}{4} \lambda \eta^{4}-\lambda v \eta^{3}-\frac{1}{4} \mathcal{F}_{\mu \nu}^{a} \mathcal{F}_{a}^{\mu \nu} .
$$


Compared to global symmetry breaking, the mass term in this last expression has an opposite sign to that in (4.13), while the other two field components have been gauged out of the theory. Furthermore, equation (4.20) shows that $A^{1}$ and $A^{2}$ have acquired a mass of $g^{2} v^{2}$. This can be seen more clearly from the Euler-Lagrange equations

$$
\begin{gathered}
\partial_{\mu} \partial^{\mu} \eta-\left(\frac{g^{2}}{4}\right)\left(\left(A_{\mu}^{1}\right)^{2}+\left(A_{\mu}^{2}\right)^{2}\right)(\eta+v)-2 \mu^{2} \eta+\lambda\left(\eta^{2}\right) \eta+3 \lambda v \eta^{2}=0 \\
\partial_{\mu} \mathcal{F}_{a}^{\mu \nu}-\left(\frac{g^{2}}{4}\right)(\eta+v)^{2} A_{a}^{\nu}-i g\left(\tau_{a}\right)_{b c} A_{\mu}^{b} \mathcal{F}_{c}^{\mu \nu}=0, \quad \text { for } a=1,2
\end{gathered}
$$

and

$$
\partial_{\mu} \mathcal{F}_{3}^{\mu \nu}-i g\left(\tau_{3}\right)_{i j} A_{\mu}^{i} \mathcal{F}_{j}^{\mu \nu}=0
$$

The remaining local $\mathrm{SO}(2)$ invariance about $\phi_{0}$ produces a single massless gauge field that is identified with the electromagnetic field discussed in Section 2. Under an infinitesimal rotation about the third axis of $R$, the field in (4.16) transforms as

$$
\phi(x) \longrightarrow \phi(x)
$$

while the gauge fields transform according to

$$
A_{\mu}^{j} \longrightarrow A_{\mu}^{j}+i g \theta^{3}\left(\tau_{3}\right)_{k}^{j} A_{m}^{k} u
$$

Thus, the transformation parameters are

$$
\begin{aligned}
& \Omega_{a}^{i}=0, \quad \text { for } a=1,2,3 \\
& \Omega_{a \nu}^{j}=0, \quad \text { for } a=1,2 \\
& \Omega_{3 \nu}^{j}=i g\left(\tau_{3}\right)_{k}^{j} A_{\nu}^{k},
\end{aligned}
$$

so that the conserved current associated with this symmetry is

$$
J_{3}^{\mu}=-i g\left(\tau_{3}\right)_{2}^{1}\left\{\mathcal{F}_{1}^{\mu \nu} A_{\nu}^{2}-\mathcal{F}_{2}^{\mu \nu} A_{\nu}^{1}\right\}
$$

Comparison to equation (4.23) shows that this current coincides with the source for the electromagnetic field:

$$
\partial_{\mu} \mathcal{F}_{3}^{\mu \nu}=J_{3}^{\nu}
$$




\section{Finite Energy Solutions and the t'Hooft-Polyakov Monopole}

In the last section, the local $\mathrm{SO}(3)$ symmetry was broken down to a $\mathrm{U}(1)$ invariance. The resulting theory described the interaction of a massive charged scalar field and three gauge fields, two of which were seen to acquire mass. Another interesting feature of the $\mathrm{SO}(3)$ symmetry breaking is the existence of distinct finite energy solutions such that the transition from one to the other would require an infinite amount of energy. One such state was the lowest energy configuration discussed in the preceding section. Another such state is the t'Hooft-Polyakov monopole which will be treated in this section.

The Lagrangian density for the local $\mathrm{SO}(3)$ gauge theory with $\mu^{2}<0$ was given by

$$
\mathcal{L}=\frac{1}{2}\left(D^{\mu} \phi\right)^{i}\left(D_{\mu} \phi\right)^{i}-\frac{\lambda}{4}\left(\|\phi\|^{2}-v^{2}\right)^{2}-\frac{1}{4} F_{\mu \nu}^{a} F^{a, \mu \nu}
$$

where we have used the adjusted potential so that the vacuum configuration yields zero total energy. In terms of the quantities appearing in (5.1) this energy is given by

$$
E=\int d^{3} x\left[\frac{1}{2}\left(D_{\mu} \phi\right)^{i}\left(D^{\mu} \phi\right)^{i}+\frac{1}{4} \lambda\left(\|\phi\|^{2}-v^{2}\right)^{2}+\frac{1}{4} \mathcal{F}_{\mu \nu}^{a} \mathcal{F}^{a, \mu \nu}\right]
$$

A necessary condition for this integral to yield finite energy is that the field configuration asymptotically satisfy $\|\phi\| \rightarrow v$ as $r \rightarrow \infty$. Other necessary restrictions on the remaining terms in (5.2) require that both quadratic terms, involving the covariant derivatives and the field strength, behave like $1 / r^{4}$ asymptotically. [2] The configuration that will be considered in this section is one for which the scalar field is radial for large values of $r$ :

$$
\phi^{i}(\mathrm{r}) \longrightarrow v \hat{r}^{i} \quad \text { as } \quad r \rightarrow \infty,
$$


where $\hat{r}^{i}$ is the ith component of the unit vector in the direction of $\mathbf{r}$.

Two points about the requirement (5.3) need mention. The first regards the stability of this class of solutions. The fact that (5.3) is topologically distinct from the ground state $\phi_{0}$ of Section 4 can be seen by the fact that $\phi_{0}$ maps the 2-sphere at spatial infinity onto a single point of the 2 -sphere $\|\phi\|=v$, while the field given by (5.3) maps the sphere at spatial infinity to a one-time covering of the 2-sphere $\|\phi\|=v$. Just as the number of windings around a circle distinguishes different homotopy classes, these images are homotopically distinct: there is no continuous deformation of the image of(5.3) to that of $\phi_{0}$. In this topological sense, (5.3) represents the simplest possible case beyond the trivial configuration of the vacuum [3]. The second point to be made about (5.3) is that it constitutes a violation of gauge invariance [6]. The system can no longer be rotated by arbitrary $\mathrm{SO}(3)$ transformations such that this asymptotic form is preserved. Instead it retains only a $U(1)$ symmetry about the radial direction similar to the $U(1)$ invariance associated with $\phi_{0}$ found in section 4 . That is, the $\mathrm{U}(1)$ invariance of electromagnetism can be identified with these rotations about the radial direction. This will enable to determine the electromagnetic field strength after the t'Hooft-Polyakov solution is given.

The t'Hooft-Polyakov monopole is determined by adopting an "ansatz"for the particular forms of the the scalar and gauge fields [2]:

$$
\begin{aligned}
\phi^{i} & =\frac{r^{i}}{q r^{2}} f(r) \\
A_{a}^{i} & =\epsilon_{a i j} \frac{r^{j}}{q r^{2}}(1-h(r))
\end{aligned}
$$

It is further assumed that the time components of the gauge potentials are equal to zero, so that together with the time-independence of the scalar field, (5.4) forms a static field configuration. By inspecting (5.2), one can see that static solutions 
form a lower bound on the energy of the system, since the integral is positive in the quadratic terms involving the covariant derivatives. Static sable solutions also constitute a first stage from which perturbations can be made.

The fields in (5.4) depend on two functions, $f(r)$ and $h(r)$. We will now determine a pair of coupled second-order equations for these functions that have equivalent dynamical content as the field equations obtained from (1.9). To derive these equations, express each of the quantities in Lagrangian (5.1) in terms of the functions $f(r)$ and $h(r)$ found in the ansatz and then perform variations on the Lagrangian with respect to each of these functions. In order to simplify this process, first observe that the Lagrangian can be grouped into three parts: the potential $V(\phi)$, another containing only the gauge fields, $\mathcal{L}_{0}$, and the interaction term involving the covariant derivatives, $\mathcal{L}_{\text {int }}$.

$$
\mathcal{L}=\mathcal{L}_{\text {int }}+\mathcal{L}_{0}+V(\phi)
$$

The differential equation for $f(r)$ will result from the potential term and the interaction part of the Lagrangian, while the equation for $h(r)$ will be determined by a variation of the interaction part along with the free gauge field term of the Lagrangian.

The covariant derivatives are

$$
D_{0} \phi^{j}=0, \quad j=1,2,3
$$

which is consistent with the previous assumption, and

$$
D_{i} \phi^{j}=\partial_{i} \phi^{j}+A_{i}^{a}\left(T_{a}\right)_{k}^{j} \phi^{k}=\partial_{j}\left(\frac{r^{i}}{r^{2}} f(r)\right)+\left[\epsilon_{a i m} \frac{r^{m}}{r^{2}}(1-h(r))\right]\left(-\epsilon_{a j k} \frac{r^{k}}{r^{2}} f(r)\right)
$$

or

$$
D_{i} \phi^{j}=\left(\frac{f(r)}{r^{2}}\right)^{\prime} \frac{r^{i} r^{j}}{r^{2}}+\frac{f(r)}{r^{2}} \delta_{i j}-\left(\frac{(1-h(r))}{r^{2}}\right)\left(\frac{f(r)}{r^{2}}\right)\left(\delta_{i j} r^{2}-r^{i} r^{j}\right) .
$$


Hence, the interaction term in (5.1) is

$$
\begin{aligned}
\left(D_{j} \phi\right)^{i}\left(D^{j} \phi\right)^{i} & =r^{2}\left[\left(\frac{f(r)}{r^{2}}\right)^{\prime}\right]^{2}+2 r\left(\frac{f(r)}{r^{2}}\right)^{\prime}\left(\frac{f(r)}{r^{2}}\right)+3\left(\frac{f(r)}{r^{2}}\right)^{2} \\
& +2\left(\frac{f(r)}{r^{2}}\right)^{2}(1-h(r))^{2}-4\left(\frac{f(r)}{r^{2}}\right)^{2}(1-h(r)) \\
& =r^{2}\left[\left(\frac{f(r)}{r^{2}}\right)^{\prime}\right]^{2}+2 r\left(\frac{f(r)}{r^{2}}\right)^{\prime}\left(\frac{f(r)}{r^{2}}\right)+\left(\frac{f(r)}{r^{2}}\right)^{2}\left(1+2 h^{2}\right) .
\end{aligned}
$$

If the derivative

$$
\left(\frac{f(r)}{r^{2}}\right)^{\prime}=\frac{f^{\prime}(r)}{r^{2}}-2 \frac{f(r)}{r^{3}}
$$

is inserted in this last expression, then, after some algebra, the product (5.9) reduces to

$$
\left(D_{j} \phi\right)^{i}\left(D^{j} \phi\right)^{i}=\frac{\left(f^{\prime}(r)\right)^{2}}{r^{2}}-2 \frac{f(r) f^{\prime}(r)}{r^{3}}+\frac{(f(r))^{2}}{r^{4}}\left(2(h(r))^{2}+1\right) .
$$

The potential is easily computed. From (4.6), we have

$$
V(\phi)=\frac{1}{4} \lambda\left(\|\phi\|^{2}-v^{2}\right)^{2}=\frac{1}{4} \lambda\left(\frac{r^{2}(f(r))^{2}}{r^{4}}-v^{2}\right)^{2} .
$$

The differential equation for $f(r)$ can now be found by varying

$$
\begin{aligned}
L_{1}(f(r))=\int d x^{3}\{ & \frac{1}{2} \frac{\left(f^{\prime}(r)\right)^{2}}{r^{2}}-\frac{f(r) f^{\prime}(r)}{r^{3}}+\frac{1}{2} \frac{(f(r))^{2}}{r^{4}} \\
& \left.+(h(r))^{2} \frac{(f(r))^{2}}{r^{4}}-\frac{1}{4} \lambda\left(\frac{r^{2}(f(r))^{2}}{r^{4}}-v^{2}\right)^{2}\right\}
\end{aligned}
$$

with respect to $f$. This volume integral reduces to

$$
\begin{aligned}
L_{1}=4 \pi \int_{0}^{\infty} d r\{ & \frac{1}{2}\left(f^{\prime}(r)\right)^{2}-\frac{1}{r} f(r) f^{\prime}(r)+\frac{1}{2 r^{2}}(f(r))^{2} \\
& \left.+\frac{1}{r^{2}}(h(r))^{2}(f(r))^{2}-\frac{1}{4} \lambda r^{2}\left(\frac{r^{2}(f(r))^{2}}{r^{4}}-v^{2}\right)^{2}\right\}
\end{aligned}
$$

in spherical coordinates. Varying this integral, the corresponding Euler-Lagrange equation for $f$ is

$$
r^{2} \frac{d^{2} f}{d r^{2}}=f\left[2 h^{2}-\mu^{2} r^{2}+\frac{\lambda}{g^{2}} f^{2}\right]
$$


The second equation can be found by calculating the product $\mathcal{F}_{\mu \nu}^{a} \mathcal{F}^{a, \mu \nu}$, where, again, $\mathcal{F}_{\mu \nu}^{a}$ is given by

$$
\mathcal{F}_{i j}^{a}=\partial_{i} A_{j}^{a}-\partial_{j} A_{i}^{a}+q \epsilon_{a b c} A_{i}^{b} A_{j}^{c} .
$$

The product is

$$
\mathcal{F}_{i j}^{a} \mathcal{F}^{a, i j}=\left(\partial_{i} A_{j}^{a}-\partial_{j} A_{i}^{a}+q \epsilon_{a b c} A_{i}^{b} A_{j}^{c}\right)\left(\partial_{i} A_{j}^{a}-\partial_{j} A_{i}^{a}+q \epsilon_{a b^{\prime} c^{\prime}} A_{i}^{b^{\prime}} A_{j}^{c^{\prime}}\right)
$$

Thus, the field strength term in (5.1) becomes

$$
\begin{aligned}
-\frac{1}{4} \mathcal{F}_{i j}^{a} \mathcal{F}^{a, i j}=-\frac{1}{2} r^{4}\left(\frac{1-h}{r^{2}}\right)^{4} & +2 r^{2}\left(\frac{1-h}{r^{2}}\right)^{3}-6\left(\frac{1-h}{r^{2}}\right)^{2} \\
& -r^{2}\left(\left(\frac{1-h}{r^{2}}\right)^{\prime}\right)^{2}-4 r\left(\frac{1-h}{r^{2}}\right)^{\prime}\left(\frac{1-h}{r^{2}}\right) .
\end{aligned}
$$

When the derivative

$$
\left(\frac{1-h}{r^{2}}\right)^{\prime}=-\frac{h^{\prime}}{r^{2}}-2 \frac{(1-h)}{r^{3}}
$$

is inserted, (5.18) simplifies to

$$
\mathcal{L}_{0}=-\frac{1}{2} \frac{(1-h)^{4}}{r^{4}}+2 \frac{(1-h)^{3}}{r^{4}}-2 \frac{(1-h)^{2}}{r^{4}}-\frac{\left(h^{\prime}\right)^{2}}{r^{2}}
$$

or

$$
\mathcal{L}_{0}=-\frac{1}{2} \frac{1}{r^{4}}\left[\left(h^{2}-1\right)^{2}\right]-\frac{\left(h^{\prime}\right)^{2}}{r^{2}} .
$$

The action integral for $\mathrm{h}$ follows from combining equation (5.17) with the interaction term (5.9):

$$
\begin{aligned}
L_{2}(h)=\int d^{3} x\left\{\mathcal{L}_{i n t}\right. & \left.+\mathcal{L}_{0}\right\} \\
=4 \pi \int_{0}^{\infty} d r\left\{\frac{1}{2}\left(f^{\prime}\right)^{2}-\frac{1}{r} f f^{\prime}+\frac{1}{2 r^{2}}(f)^{2}\right. & \\
& \left.\quad+\frac{1}{r^{2}}(h)^{2}(f)^{2}-\frac{1}{2} \frac{1}{r^{4}}\left[\left(h^{2}-1\right)^{2}\right]-\frac{\left(h^{\prime}\right)^{2}}{r^{2}}\right\} .
\end{aligned}
$$


Varying this last expression with respect to $h$, the differential equation for $h$ is found to be

$$
r^{2} \frac{d^{2} h}{d r^{2}}=h\left[(h)^{2}+(f)^{2}-1\right]
$$

Furthermore, from equations (5.11), (5.12), and (5.21), the energy (5.2) can expressed in terms of these functions as

$$
E=\frac{4 \pi}{g^{2}} \int_{0}^{\infty} d r \frac{1}{r^{2}}\left[\frac{1}{2}\left(r f^{\prime}-f\right)^{2}+f^{2} h^{2}+\frac{\lambda}{4 g^{2}}\left(f^{2}-(g v r)^{2}\right)^{2}+r^{2}\left(h^{\prime}\right)^{2}+\frac{1}{2}(h-1)^{2}\right]
$$

Following Cheng and $\mathrm{Li}[]$, we simplify this expression by introducing the notation $\xi \equiv g v r$ and express the energy as

$$
E=\frac{4 \pi v}{g} \int_{0}^{\infty} d \xi \frac{1}{\xi^{2}}\left\{\frac{1}{2}\left(\xi \frac{d f}{d \xi}-f\right)^{2}+f^{2} h^{2}+\frac{\lambda}{4 g^{2}}\left(f^{2}-(\xi)^{2}\right)^{2}+\xi^{2}\left(\frac{d h}{d \xi}\right)^{2}+\frac{1}{2}(h-1)^{2}\right\}
$$

Now, the asymptotic condition (5.3) implies that the function $f$ appearing in (5.4) must satisfy $f \rightarrow q v r$ as $r \rightarrow \infty$. For the integral in (5.25) to converge, further necessary conditions are imposed on the functions $f$ and $h$ : $h$ vanishes asymptotically and both $\mathrm{f}$ and 1 - $\mathrm{h}$ are bounded and are of the same order of magnitude or less as $r$ near $r=0$ [2]. Solutions to (5.15) and (5.23) satisfying these requirements exist and their behavior is indicated by the following diagram:

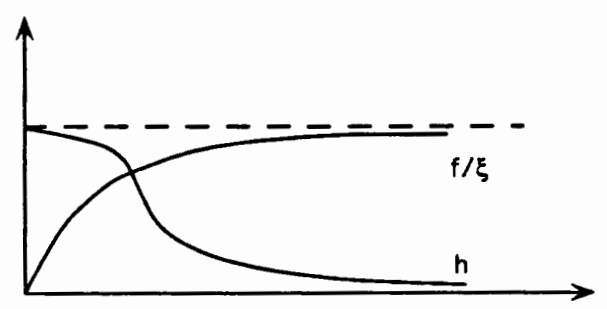

The graph indicates that the scalar field in (5.4) is close to zero near $r \simeq 0$ and grows linearly away from zero. It also shows that $\phi$ satisfies the boundary condition 
(5.3). Secondly, since 1 - $\mathrm{h}$ does not differ appreciably from zero on some compact set near $r=0$, the gauge fields are roughly constant and close to zero in this region. Furthermore,

$$
A_{a}^{i} \longrightarrow \epsilon_{a i k} \frac{r^{k}}{q r^{2}}, \quad \text { as } r \longrightarrow \infty
$$

as can be seen from the behavior of $h$. Thus, the gauge fields behave in a way consistent with the comments directly preceding (5.3). From (5.26), the asymptotic form of the field strength can be computed. The derivative terms in (5.16) cancel:

$$
\partial_{i} A_{j}^{a}-\partial_{j} A_{i}^{a}=\frac{1}{q r^{2}}\left[\epsilon_{a i j}+\frac{2}{r^{2}}\left(\epsilon_{a j k} r^{i} r^{k}-\epsilon_{a i k} r^{j} r^{k}\right)\right],
$$

as can be seen by considering different values for $a, i$, and $j$. Then, for large values of $r$, only the quadratic term in (5.16) survives, so that the field strengths have the form

$$
\mathcal{F}_{a}^{i j}=-q \varepsilon_{a b c} A_{i}^{b} A_{j}^{c}=-q \varepsilon_{a b c} \varepsilon_{b i k} \varepsilon_{c j l} \frac{1}{q^{2} r^{4}} r^{k} r^{l}
$$

where the asymptotic value for A in (5.4) has been used. Invoking (5.4) once more, the field strengths can be expressed in terms of the scalar field at large distances:

$$
\mathcal{F}_{a}^{i j}=\frac{1}{q r^{4}} \varepsilon_{i j k} r^{k} r^{a}=\varepsilon_{i j k} \frac{r^{k}}{q v r^{3}} \phi^{a},
$$

Recalling the discussion following (5.3), the electromagnetic field strength is identified with the $U(1)$ symmetry possessed by the system at large distances about the radial direction. The expression (5.29) for the various field strengths involved is given in terms of the standard axes in the internal space associated with the scalar field. The radial part of the field strength is obtained by projecting (5.29) along the radial direction. That is,

$$
\mathcal{F}_{i j} \equiv \mathcal{F}_{i j}^{a} \hat{r}^{a}
$$


where $\hat{r}^{a}=\frac{\phi^{a}}{\|\phi\|}$. Therefore,

$$
\mathcal{F}_{i j}=\varepsilon_{i j k} \frac{r^{k}}{q r^{3}}=\varepsilon_{i j k} \frac{1}{q r^{2}} \hat{r}^{k}
$$

Thus, the electromagnetic field strength only contains magnetic components. The resulting field is

$$
\mathrm{B}(r)=\frac{\mathbf{r}}{q r^{3}}
$$

which is a configuration due to a magnetic pole of charge

$$
\int_{\Sigma} \mathbf{B} \cdot d \mathbf{S}=\frac{4 \pi}{q}
$$

where $\Sigma$ is a simple closed surface containing the origin. Note that when Planck's constant $\hbar$ and $c$ are set equal to one, both (5.32) and (5.33) agree with Dirac's monopole for $n=2$ (compare to (1.2)).

The question of the size of the monopole can now be addressed. The differential equations, (5.15) and (5.23), for $f$ and $h$

respectively, simplify to

$$
\frac{d^{2} h}{d \xi^{2}}=h, \quad \frac{d^{2} \tilde{f}}{d \xi^{2}}=\frac{2 \lambda}{q^{2}} \tilde{f}
$$

in the limit $r \rightarrow \infty$, where the variable $\xi=q v r$ and $\tilde{f}=f-\xi$ [2]. Well-behaved solutions to (5.34) for large $\xi$ are easily found:

$$
\begin{gathered}
h(r) \sim e^{-q v r}=e^{-M r} \\
f(r)-\xi \sim \exp \left\{-\sqrt{2 \lambda} \frac{\xi}{q}\right\}=\exp \left\{-\sqrt{-2 \mu^{2}} r\right\} .
\end{gathered}
$$

where $\alpha^{2}=-2 \mu^{2}$ and $\mathrm{M}$ are the masses of the scalar field and the massive vector fields found in section 4. As is generally observed, how rapidly the t'Hooft-Polyakov 
solution approaches the monopole field configuration (5.32) is determined solely by the masses that were acquired by the various fields when the $\mathrm{SO}(3)$ symmetry was spontaneously broken down to $\mathrm{U}(1)$ [2]. The monopole is confined to a region $r<\alpha^{-1}$ or $r<M^{-1}$, whichever is greater. The fields outside this region conjoin to produce a static configuration that quickly approximates the monopole field (5.32); meanwhile, within this region these fields provide the monopole with an internal structure. Moreover, since all the field components in this analysis are smooth, the Dirac string singularity does not appear in the final construction.

The energy integral (5.25) provides the rest mass $(c=1)$ of the monopole. This mass depends on the parameter $\lambda$, the coupling constant $q$, and the expectation value $v$ (i.e., $\sqrt{-\mu^{2} / \lambda}$ ). This integral does not differ substantially from $\frac{4 \pi v}{q^{2}}$ for various values of $\frac{\lambda}{q^{2}}$. Thus, the mass of the monopole is said to be determined by the scale, $\mathrm{v}$, set when the original $\mathrm{SO}(3)$ symmetry is broken down to $\mathrm{U}(1)$ in Section 4 [2]. 


\section{References :}

1. Dirac, P. A. M., Proc. Royal Soc. of London, A, 133, 1931

2. Cheng, T. and Li, L., "Gauge Theories of Elementary Particle Physics", Oxford Univ. Press, New York, 1984

3. Guidry M., "Gauge Field Theories: An Introduction with Applications", Wiley and Sons, New York, 1991

4. Jackson, J.D., "Classical Electrodynamics", Wiley and Sons, New York, 1975

5. Huang, K. "Quarks, Leptons and Gauge Fields", Cambridge Univ. Press, New York, 1987

6. Göckeler, M. and Schücker, T., "Differential Geometry, Gauge Theories, and Gravity", Cambridge Univ. Press, New York, 1987 